\title{
Saverio Muratori (1910-1973)
}

La ville comme seul modèle

Saverio Muratori (1910-1973), The City as a Model

Jean Castex

\section{(2) OpenEdition}

Journals

Édition électronique

URL : http://journals.openedition.org/crau/446

DOI : $10.4000 /$ crau. 446

ISSN : 2547-5746

Éditeur

Éditions du patrimoine

Édition imprimée

Date de publication : 1 mars 2014

Pagination : 13-35

ISBN : 978-2-7577-0359-5

ISSN : 1296-4077

Référence électronique

Jean Castex, « Saverio Muratori (1910-1973) », Les Cahiers de la recherche architecturale et urbaine [En ligne], 29 | 2014, mis en ligne le 13 septembre 2017, consulté le 01 mai 2019. URL : http://

journals.openedition.org/crau/446 ; DOI : 10.4000/crau.446 
Le centenaire de la naissance de Muratori (1910-1973) a été l'occasion de mieux comprendre comment l'un des initiateurs de la morphologie urbaine concevait la ville. Revenant aux sources qui ont guidés ses propres recherches sur « la ville comme seul modèle ", en liaison avec Philippe Panerai, I'auteur fait un double point sur la carrière de Muratori. II montre sa participation aux chantiers INA-Casa à Rome et son échec pour le quartier neuf des Barene di San Giuliano, face à Venise, qui sert de modèle (1949-1959). Puis il généralise ses conceptions en faisant appel à un demi-siècle pendant lequel l'école muratorienne a prouvé ses capacités d'intelligence de la ville et du territoire. Quatre éléments fondateurs charpentent les objectifs de Muratori : le type comme concept actif ou l'adaptation graduelle de la ville; la série des échelles ; la storia operante; le tissu banal de la ville.

\section{Saverio Muratori (1910-1973)} La ville comme seul modèle
Comprendre la morphologie urbaine selon Saverio Muratori caractérise une méthode où l'analyse s'articule indissolublement au projet qui la met à l'épreuve. Ce travail postule la continuité historique du passé et du présent. Nous évoquerons plus particulièrement Muratori ${ }^{1}$ autour de deux périodes: les constructions de I'INA-Casa à Rome entre 1949 et 1954, puis le concours des Barene di San Giuliano face à Venise de 1959, qui a provoqué un drame par le refus des architectes "modernes» de tenir compte de la science de la ville - la " vraie ville ${ }^{2} »-$ que défendait Muratori. Son côté « innovant et d'avant-garde », pour reprendre les propos de Giancarlo Cataldi ${ }^{3}$, a secoué la culture de l'architecture en Italie par l'opposition des "grands maîtres " de l'architecture et passe "comme un événement ignoble " qui entache cette culture (la phrase est encore de Cataldi). Toutefois, j'aurai à déborder la période de 1949-1959, pour en couvrir une plus large, de 1932 à 1989, seize ans après sa mort.

1 Marina Montuori avait rédigé une note biographique dans Lezioni di progettazione, Milan, Electa, 1988, p. 130-131. L'ouvrage, dont les pages 130 à 161 sont consacrées à Muratori, comprend une introduction de Francesco Tandori, p. 7-13, qui souligne les convictions abstraites de Muratori d'avant la guerre, laissant espérer un brillant avenir quand I'Italie fut libérée du fascisme. Franco Purini ("La scuola romana dagli anni sessanta agli anni ottanta », p. 272-295) révèle sa rigueur qui conduisit permit de comprendre la ville en donnant des modèles. Son arrivée à Venise est due à la volonté de Guiseppe Samonà (1898-1983) de réorganiser le corps enseignant de la faculté d'architecture, désorienté, pour en faire le lieu d'oppositions entre adversaires souvent acharnés. Mais c'est bien Venise qui lui permit de développer la connaissance du processus de croissance de la ville. à une sorte d'anti-historicisme : elle lui 


\section{L'imprégnation de l'après-guerre à Rome Les chantiers de I'INA-Casa}

La situation de I'Italie, après le fascisme, provoque la renaissance d'un débat un long moment repoussé: l'incapacité de Mussolini à résorber les poches de pauvreté oblige le président Fanfani à proposer la construction de logements populaires, pour la durée de deux septennats, de 1949 à 1963, sous le nom d'INA-Casa (Institut national de l'assurance pour la maison). Créer des quartiers neufs infléchit les positions doctrinales et ouvre aux notions de type, de ville et de territoire. Muratori est impliqué dans les réalisations d'INA-Casa à Rome ${ }^{4}$, il coordonne avec Mario de Renzi (1897-1967) deux opérations: Valco San Paolo (juin-septembre 1949-1950) pour 440 logements et 2600 habitants, et Tuscolano II (1950-1952), 3150 logements, 11500 habitants. Ludovico Quaroni et Mario Ridolfi de leur côté travaillent sur Tiburtino IV (août 1950-1954), 684 logements, 4000 habitants.

Trois ensembles d'idées sont communs aux opérations d'INA-Casa. II s'agit surtout de refuser la répétitivité et le caractère mécaniciste marqué par le Mouvement moderne. Le retour du vocable «spontanéité », repris de la "spontaneitet » des jeunes Scandinaves, se charge d'une réévaluation du projet technique au profit d'une culture héritée et admise: elle renverse les buts du fonctionnalisme et a l'ambition de traiter d'un habitant réel et concrètement situé. Mais c'est vers une culture populaire que tendent les efforts. L'Italie fait le choix de la décentralisation, du local, des techniques traditionnelles. Bien que contrôlée par l'État, la politique de I'INA-Casa laisse leur autonomie aux chantiers: elle
VESPAI SOLAI BALCONI - VESPAI: TIPI PRINCIP. 8a

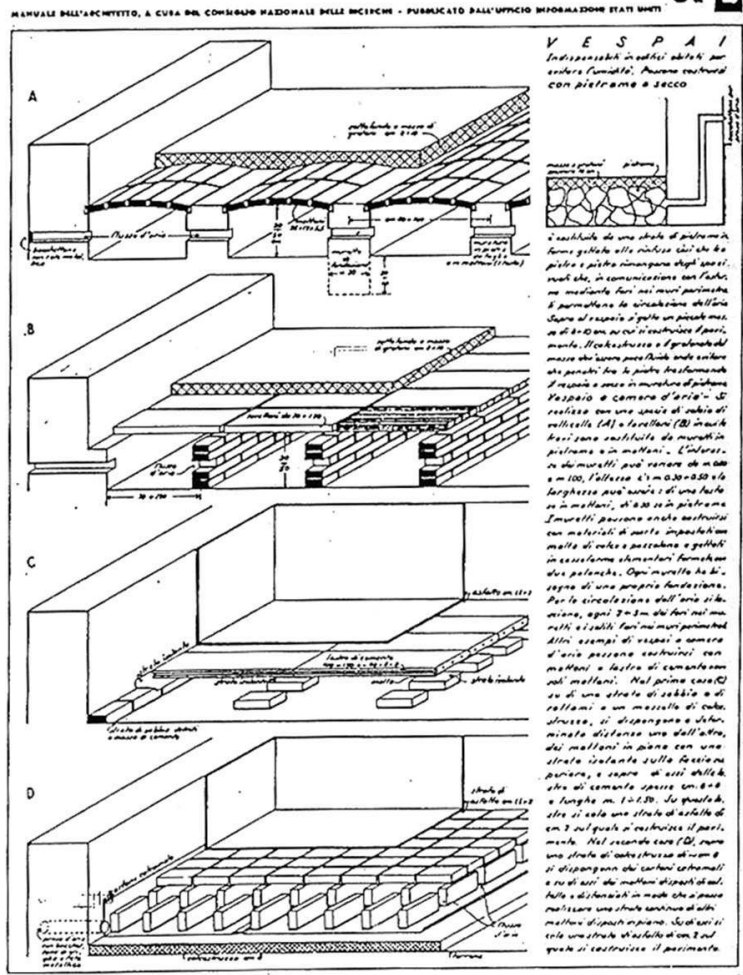

Mario Ridolfi a marqué son influence sur les opérations INA-Casa en 1950 en modernisant les techniques populaires de la construction. Il s'affranchissait du dogme de l'industrie et avait facilité l'interprétation de « la vraie ville " préconisée par Muratori.
À Rome, après l'échec de son projet pour les Barene en 1960, il devint une sorte de «banni ». II s'en releva. En 1963, après des mouvements de contestation à la faculté d'architecture, il prononça le discours de Roxi, un champ de désespoir, dirigé non contre les étudiants mais contre les fausses abstractions qui gouvernaient le Moderne et s'opposaient au sens commun des habitants. Les étudiants l'acclamèrent et il put commencer un cours nouveau en 1964-1965 en développant ses méthodes. Après

l'exposition de mai à juin 1991 tenue à Modène,
«Saverio Muratori, Architetto, Modena 1910, Roma, 1973 », une bibliographie (Bibliografia a cura della Biblioteca Civica di Storia dell'Arte " Luigi Poletti » di Modena) précise ses écrits, ceux, de caractères généraux autour de lui, ceux liés à son activité de projet, relatifs à son cours de composition architectonique, ceux de l'école muratorienne.

Cet article est en lien avec mon habilitation à diriger des recherches, Une typologie à usages multiples, classer, comprendre, projeter (sous le tutorat de François Loyer, Énsa de Versailles,
Ladrhaus, novembre 2001), comprenant 52 pages sur Muratori, avec trois volumes d'articles. Consulter Jean-Louis Cohen, « La coupure entre architectes et intellectuels, ou les enseignements de l'italophilie ", In Extenso, n' 1, 1984. Parmi de nombreux textes sur Muratori, plusieurs références ont été faites à Giorgio Pigafetta, Saverio Muratori, architetto. Teoria e projetti, Venise, Marsilio, 1990. Le texte reprend la conférence dédiée à Muratori peu de temps après le centenaire de sa naissance, faite à l'université de Delft, pour un colloque de l'ISUF 


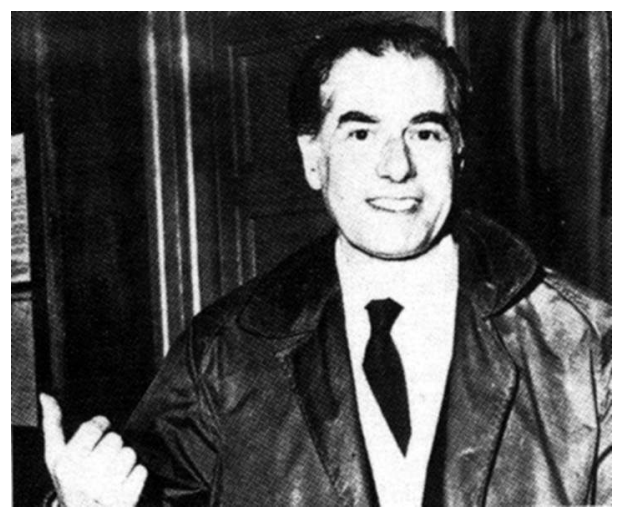

Saverio Muratori au début des années 1970.

Marina Montuori, Lezioni di progettazione : 10 maestri dell'architettura italiana, Milan, Electa, 1988, p. 130.

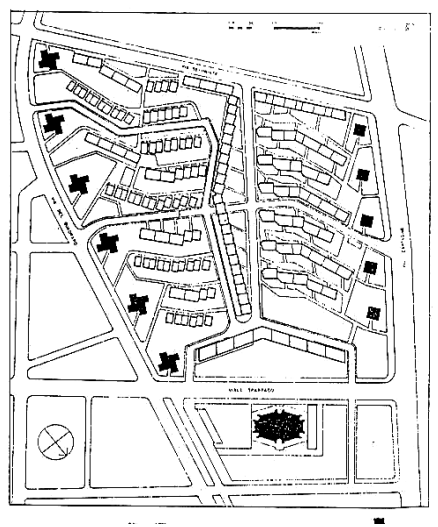

À Tuscolano II à Rome, Muratori travaille avec Mario De Renzi, Lucio Cambellotti, Giuseppe Perugini, Dante Tassotti et Luigi Vagnetti de 1951 à 1955. Le bâtiment plié face à l'église de l'Assomptionde-Marie échappe aux critiques de Caniggia qui ne voit dans le plan qu'une approche sentimentale de la ville : il définit le côté d'une place et s'insère dans le concept du tissu. se base sur la diminution du chômage qui touche de larges secteurs de la population ${ }^{5}$. Elle a pour but de développer une culture populaire. Utilisant des ouvriers de faible formation professionnelle, elle connaît une réaction anti-technologique dont les conséquences sont essentielles. Elle doit rassembler les cultures locales du bâtiment, avec l'objectif avoué de "définir un langage directement accessible aux classes populaires ${ }^{6} »$.

Ce retour à des technologies et des motifs traditionnels et populaires ${ }^{7}$ demandait une mise à plat complète. Elle s'accomplit en 1946 avec le Manuale dell'architetto dans lequel Mario Ridolfi ${ }^{8}$ contrôle dessins et texte. Ce manuel a connu une diffusion exceptionnelle? ${ }^{9}$ Basé sur les techniques de construction et non sur la distribution comme le Neufert, l'ouvrage s'ouvre à une mise à plat et à un début de théorie. II respecte la dissémination régionale, limite les généralisations, montre l'enracinement local des métiers mais le modernise et finit par toucher même les types de bâtiment. Dans un lieu donné il met en valeur la continuité du travail: Muratori y sera sensible dans le chantier de Tuscolano II, au point de faire droit aux " phénomènes de construction spontanés » qui prendront place dans sa théorie.

Quaroni et Ridolfi conscients de la présence de la culture artisanale comprennent que la position de I'architecte doit se modifier. Elle lui imprime, malgré ses compétences, une modestie visible sur le chantier. Muratori bataillera contre l'individualisme en l'accusant de faire sortir le projet de "la convention» qui doit le discipliner: il finira par développer un «magistère austère ", à force des retraits qu'elle exigeait. Ridolfi se transforme en «maître maçon » du projet, donnant
(International Seminar on Urban Form), donnée le 17 octobre 2012. La faculté d'architecture de l'université de Delft avaient traduit le texte en anglais, légèrement différent du texte en français, avec la compétence (et les critiques) de Kevin Cook et de Susanna Komossa. R. Cavallo, S. Komossa, J. Kuyper, M. Marzot (dir.), New Urban Configurations. Conference Proceedings, Amsterdam, EAAE / ISUF, 2014.

2. Comprendre la structure et les modes de croissance de la « vraie ville » s'oppose à
I'« abstraction » des tenants de Team $X$ et à la fabrication de termes artificiels d'analyse et de projet. Je dois à Philippe Panerai ces certitudes qu'il a brillamment exposées dans de nombreuses recherches sur les Formes Urbaines : de l'îlot à la barre, Paris, 1977 (réédité en 1997, Marseille, Éditions Parenthèses) en les poursuivant sur le Moyen-Orient et le Brésil.

3. Giancarlo Cataldi, « Design in Stages. Theory and Design in the Typological
Concept of the Italian School of Saverio Muratori ", dans Attilio Petruciolli, Typological Process and Design Theory, Cambridge (MA), Akpia, Harvard University, Massachussets Institute of Technology, 1998, p. 35-55.

4. Une analyse de trois quartiers INA-Casa a été faite à l'Énsa de Versailles, sous la direction d'Anne-Marie Châtelet, par A.-S. Auburtin, M. Dana, A. Hallé, Trois Quartiers réalisés par I'INA-Casa après la Seconde Guerre mondiale, Valco San Paolo, Tuscolano, 
Texture du bâtiment plié de Muratori à

Tuscolano II : il suit Mario Ridolfi en modernisant les techniques traditionnelles. Photographie de l'auteur.

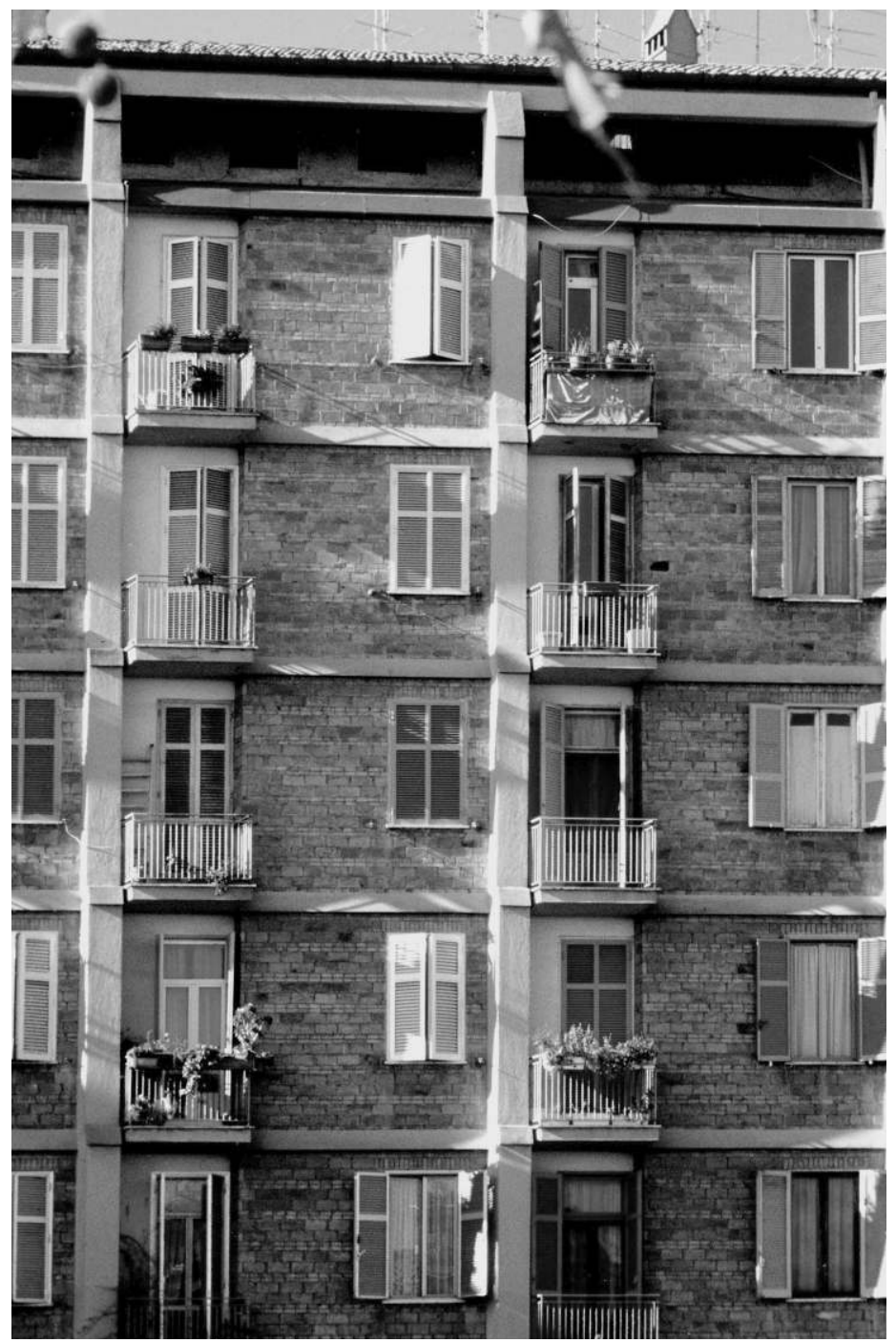

Tiburtino, Versailles, Énsa de Versailles, 1999. 5. Voir «INA-Casa al IV Congreso nazionale di Urbanistica », Venise, octobre 1952.

6. Manfredo Tafuri, Francesco Dal Co, Architecture contemporaine, Paris, Berget-Levrault, 1982.

7. Les budgets étaient limités, mais Leonardo Benevolo dans L'architettura delle città nell'Italia contemporanea, Bari, Laterza, 1972, p. 150, devra reconnaitre que INA-Casa voulait bien dire « faire vite et bien » et que le niveau de construction publique venait d'être mené « au maximum absolu de ce que l'architecture italienne [était] capable d'atteindre ».

8. Voir Cesare de Seta, L'Architettura del Novecento, Turin, Garzanti, 1981 et 1992 . "Il Manuale dell'architetto, e le prime opere del dopoguerra », p. 102-107.

9. Jean-Louis Cohen (" La coupure entre architectes et intellectuels, ou les enseignements de l'italophilie", In Extenso, n 1, Paris, 1984) a consacré une étude à Quaroni et à Ridolfi. Mario Ridolfi, C. Calpranica, A. Cardelli, M. Fiorentino éditent le Manuale dell'architetto, Rome, Consiglio Nationale delle Ricerche, USIS, 1946.

10. Cette norme s'appelle Suggerimenti, esempli e norme per la projettazione urbanistica.

11. Gianfranco Caniggia, « Saverio Muratori, 
l'impression de retourner au Moyen Âge en refusant la division entre l'architecte et les gens du chantier. II milite pour la sobriété, se passionne pour la mise en œuvre, fait reconnaître sa position "éminente " par Quaroni. Muratori suivra ce repositionnement. La politique de I'INA-Casa avait fait le choix de s'opposer aux grandes réalisations de machines à habiter du Mouvement moderne. La norme urbanistique de 1950 prévoyait "des compositions urbaines variées, articulées, capables de créer des milieux accueillants et reposants, avec des vues variées dans toutes les directions » pour procurer une "physionomie propre ${ }^{10}$. Muratori a participé à sa rédaction, refusant la rigidité des projets rectilignes, il connaissait les sources scandinaves si I'on situe en 1938 sa période " asplundienne »: c'était sans doute un moyen de ne pas se couper de la culture européenne en plein fascisme en lui offrant une solution brillante pour réintroduire la modernité sous les traits d'Asplund dans le projet du quartier de l'Eur.

Gianfranco Caniggia (1933-1987), dont le rôle est essentiel pour développer la pensée de Muratori après sa mort en 1973, s'est senti obligé de critiquer les chantiers d'INA-Casa ${ }^{11}$. Cet essai de ville n'est pas la ville. L'usage de types divers n'est pour Caniggia $q^{\prime} u^{\prime}$ " une contrefaçon ${ }^{12}$ », un souci de composition basé sur la différence, un souci de créer la variété. II touche le fond du problème : le vide entre les bâtiments n'a pas encore trouvé son sens, il reste vacant, il suit la leçon du Mouvement moderne de ne bâtir que des objets isolés dans un espace indéterminé. II fait appel à " I'area di pertinenza ${ }^{13}$ " pour bien faire comprendre que tout élément bâti s'associe à un lieu ouvert qu'il détermine: cette notion est la base du concept de ville. En l'absence d'une division en parcelles qu'on ne trouve qu'à Tiburtino IV, l'appropriation par les habitants des espaces extérieurs est pauvre et ne fournit aucun sens. L'intérêt pour la ville reste sentimental ${ }^{14}$; la prendre comme modèle exige d'abord d'en démonter la structure. Travailler sur un quartier ne dit rien sur la croissance de la ville et sur les moyens de la diriger.

Le plan de Tuscolano II reprend la forme « organique » d'un squelette. Muratori n'y réalise que le bâtiment plié sur la via Spartaco, long de 170 mètres, haut de cinq étages, soit 20 mètres. Ce n'est qu'une barre mais elle délimite une place, avec des boutiques et un cinéma, et fait face à une église " baroquisante ». L'Assunzione di Maria Santissima n'a vu que sa crypte érigée de 1954 à 1970. Trois tendances s'affrontent chez Muratori. II ne redoute pas, malgré l'usage du béton, d'être appelé barocchetto ${ }^{15}$, par une référence commune des années 1920 chez De Renzi et Ridolfi. Cet exercice de retour au passé ancre le projet dans le temps écoulé et questionne I'histoire en la mobilisant: le dôme de l'église inverse la perspective borrominienne. Surtout, il a déjà développé des analyses précises sur le découpage urbain. Francesco Dal Co ${ }^{16}$ apporte une appréciation élogieuse sur la texture de la façade de la via Spartaco: I'ossature saillante contredit la planéité du bâtiment où les logements se découpent en biais, elle suscite de l'irrégularité couplée d'«une puissante tension figurative ». II donne une réponse valable aux «rapports entre modularité, série et types ": le bâtiment par sa dimension historique a la capacité «de devenir urbain». Dal Co sait déceler
La dittatica e il pensiero » dans M. Montuori (dir.), Lezioni di progettazione, op. cit. note 1, p. $160-161$.

12. Ibid. Caniggia précise page 160 : «La varietà tipologica è una contraffazione ».

13. Sylvain Malfroy, dans le commentaire de Lecture de Florence de Caniggia, précise cette notion : "Elle attire simplement l'attention sur la complémentarité structurelle qui unit la partie construite et la partie non construite d'une disposition typologique à l'intérieur du cadre bâti de la parcelle. »

14. G. Caniggia, « Saverio Muratori, La didattica e il pensiero » dans M. Montuori (dir.), Lezioni di progettazione, op. cit. note 1.

15. Le «Barocchetto » désigne un mouvement de l'architecture à Rome qui se développe pendant les années 1920. Ce terme de dépréciation est commenté avec un sens de l'ironie le grand baroque du XVIII et du
XVIII ${ }^{e}$ siècle, en le laïcisant. II joue avec la masse qu'il oppose au jeu savant de la lumière. II fait des clins d'œil aux exemples antiques. Voir Giuseppe Strappa, « La continuità con la tradizione dell'edilizie romana del'900», dans G. Strappa (dir.), Tradizione e innovazione nell'architettura di Roma Capitale, 1870-1930, Rome, Kappa, 1989, p. 45-62.

16. Francesco Dal Co, Storia dell'architettura italiana. II Secondo Novecento, Milan, Mondadori Electa, 1997, p. 191. 
I'annonce des deux ouvrages de Muratori sur Venise et sur Rome ${ }^{17}$, intitulés de la même manière, Studi per una operante storia urbana de Venise en 1960 et de Rome en 1963 qui en fourniront la clé. Muratori s'emploie désormais à bousculer ses réticences. II remporte le concours pour la chaire universitaire de Venise en 1950. II y restera jusqu'en 1954. II s'apprête à développer ses intuitions pour comprendre le devenir de cette ville fascinante.

\section{Le concours des Barene di San Giuliano}

En 1959, le Comité de coordination des constructions populaires (CEP) lance un concours non plus pour Venise, mais pour la terre ferme sur l'autre rive de la lagune: le quartier lacustre des Barene di San Giuliano. Il faut créer un quartier résidentiel de 38,50 hectares, en l'entourant d'une zone de circulation, de commerces, de bureaux, de parties récréatives. Le but est de le relier à Mestre et au port de Marghera pour former une unité. Résidentiel ne veut pas dire quartier dortoir. L'entrée de Muratori dans la compétition révèle les particularités de son caractère. II fait preuve de «nonchalance » (Caniggia ${ }^{18}$ ), prend du retard, et au moment où ses collaborateurs croient qu'il a abandonné, il apparaît à l'agence « avec une enveloppe couverte de dessins, qui propose trois plans " (Cataldi $\left.{ }^{19}\right)$. Chaque plan est rapidement mis à l'étude par les assistants, et rendu non pas comme trois projets, mais comme un seul divisé en trois phases, avec un titre unique, "Estuario » et des chiffres romains de I à III. « Estuario III » remporte le premier prix. «Estuario I » reçoit une mention honorable.
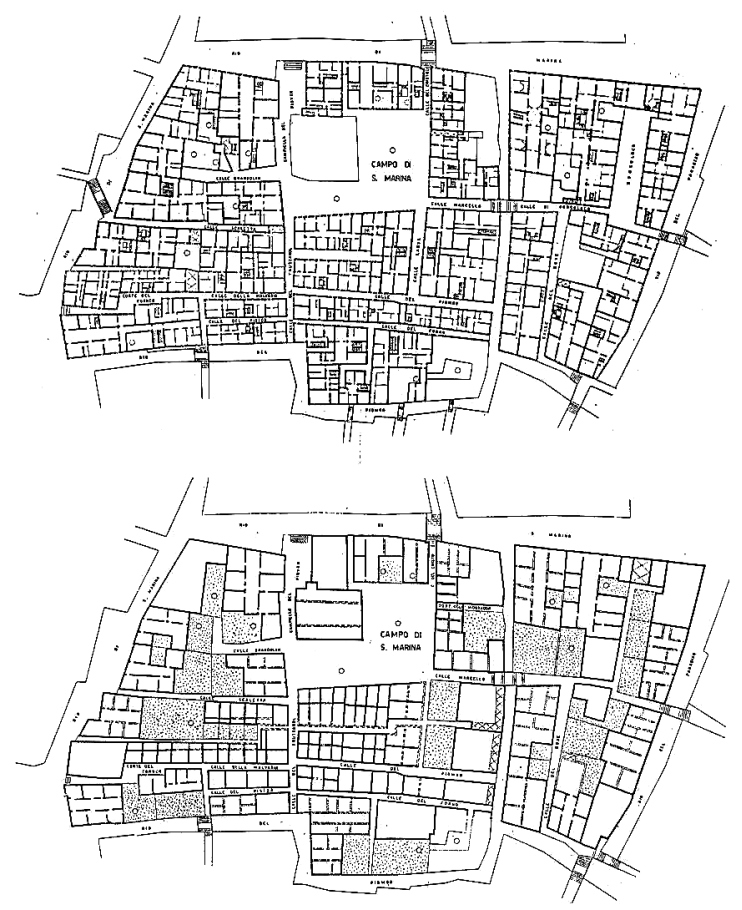

"Le Campo S

Marina, comment le relevé actuel permet de découvrir la structure d'origine ". Studi per una operante storia urbana di Venezia, 1960.

Collection de Philippe Panerai.

17. Saverio Muratori, « Studi per una operante storia urbana di Venezia », Rome, Instituto Poligrafico dello Stato, 1960, déjà publié dans Palladio 3-4, juillet-décembre 1959, p. 97-209. Et Studi per una operante storia urbana di Roma (en collaboration avec S. Bollati, R. Bollati et G. Marinucci), Rome, Consiglio nazionale delle ricerche, 1963.
18. G. Caniggia, « Saverio Muratori, la didattica e il pensiero », in M. Montuori (dir.), Lezioni di progettazione, op. cit. note 1, p. 148-161.

19. G. Cataldi, «Designing in Stages. Theory and Design in the Typological Concept of the Italian School of Saverio Muratori », dans Attilio Petruccioli (dir.), Typological Process and Design Theory, Harvard et MIT, 1988, p. 35-55. 


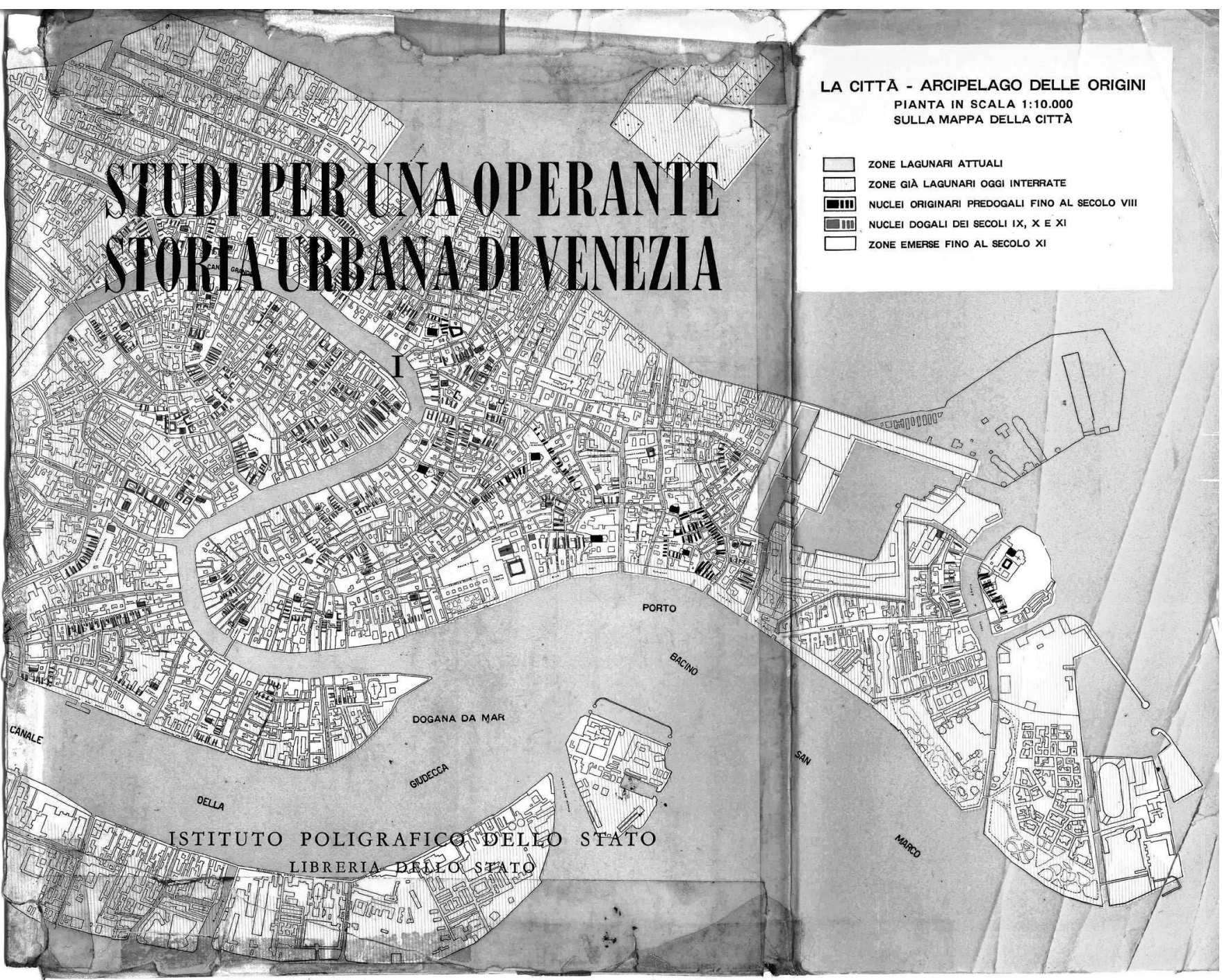

L'ouvrage de Muratori, Studi per una operante storia urbana di Venezia, 1959 et 1960 est le modèle d'une étude sur la forme de la ville.

Collection de Philippe Panerai. 


\section{La ville comme seul modèle}

De l'autre côté de la lagune envasée, les Barene di San Giuliano regardent Venise, à un peu plus de 3 kilomètres. Venise apparaît comme l'exemple à suivre ${ }^{20}$ : « Son histoire concrète et sa culture favorable à l'homme ont donné à Venise, dans le champ de la construction publique, la même excellence qu'elle procure à l'urbanisme. » Les raisons données ne sont pas formelles mais politiques - balance entre le collectif et l'individuel -, sociales - ne pas bouleverser ce qui marche-, et économiques - trouver des solutions contemporaines aux désordres graves qui menacent notre culture. Mais, comme le projet est aussi un plan de ville, il faut se garder de ne pas commettre trois types d'erreurs: sur la quantité, ne pas atteindre les limites de l'intolérable dans la dimension bâtie et la distribution; sur la série, bien voir à la fois son contenu économique et culturel; sur le style, " chasser la monotonie, le désordre, l'éphémère ». Et comme l'exigence d'un concours demande de synthétiser des règles, Muratori en propose quatre, "organisme, agrégation, types, expérimentation ». Elles sont fondées sur le passé même de Venise: un sens "sûr » de l'organisme qui contrôle la forme; un sens "vif» du groupement qui assure la solidarité et la distinction; un sens "résolu » du type; et surtout, la volonté «tenace » $d^{\prime}$ " expérimenter sur les types » ${ }^{21}$ qui a fait de Venise un lieu «toujours créateur ». Cette habile rhétorique introduit trois plans:

- Estuario I est formé d'une série d'îles « de forme, dimensions et de structures typiques, qui regroupent des grappes de quatre ou cinq éléments, liées entre elles

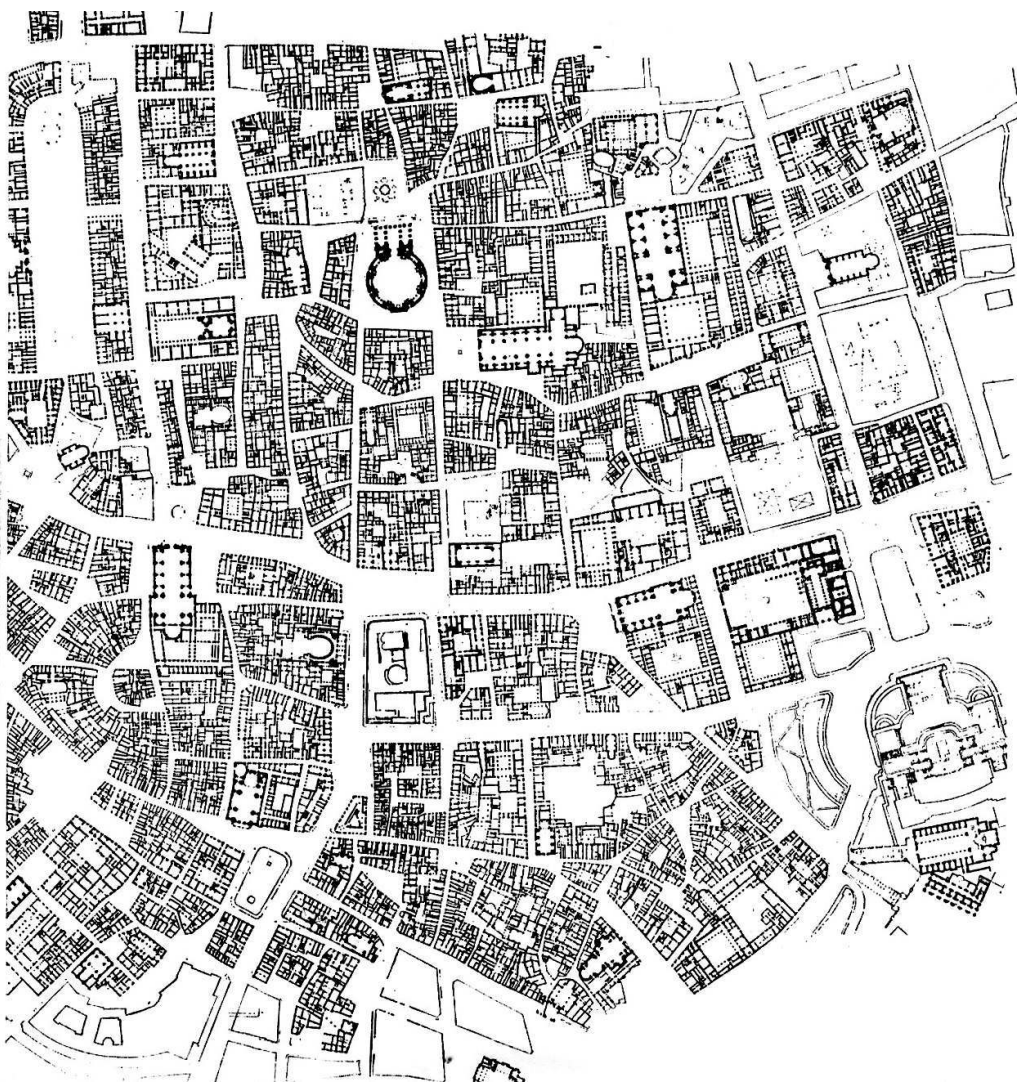

\begin{abstract}
Muratori, dans son livre Studi per una operante storia urbana di Roma, 1963, donne un relevé du centre de la ville, où l'on reconnaît la place Navone, la trace du théâtre de Pompée, le palais de Venise et le Corso, du haut à gauche suivant les aiguilles d'une montre.
\end{abstract}

20. Dans le $n^{\circ} 242$ de la revue Casabella de juillet 1960, figure la défense des architectes gagnants autour de Muratori, p. 49. Les références sont explicites: I'analyse du texte a dû être ici raccourcie.

21. Texte italien, ibid. p. 49, " La tenace sperimentazione dei tipi », plus loin "l'unità sempre creativa [...] di Venezia ». 
et à la terre ferme par des ponts et des digues », pour former des quartiers autosuffisants qui se regroupent sur la berge opposée au réseau routier. «Ce plan présente la réinterprétation par la technique et le goût moderne du schéma de la Venise de l'an mille. » Habiter sur une île accroît le contact avec le paysage, la lagune, et permet l'isolement des habitants.

- Estuario Il propose autant de péninsules pour former des quartiers autonomes selon une structure caractéristique; des canaux les séparent, mais elles sont irriguées par une voie terrestre qui gagne, au bord de la lagune, une place munie d'un équipement. Le schéma présente la forme d'un éventail, et dessine un peigne dont les dents sont parallèles au rivage. "Ce plan présente dans le goût moderne la version de la Venise gothique. "

- Estuario III se divise en deux parties appuyées aux quais (fondamenta) qui bordent l'estuaire et offrent la vue de Venise. Des îlots allongés sont délimités par deux séries de canaux, perpendiculaires et parallèles au rivage. II faut les comprendre comme l'addition des deux propositio ns précédentes, avec le choix de mieux marquer la ville comme une totalité grâce à une ligne transversale d'équipements et de services parallèle au rivage. "Ce plan s'inspire de l'urbanisme vénitien le plus récent $\left(\left.\mathrm{XVI}\right|^{\mathrm{e}}-\mathrm{xV} \|^{\mathrm{e}}\right.$ siècles), déjà moderne en sa substance. »

La ville comme elle est devient le seul modèle, toujours actif, capable de modeler son avenir. Le projet de Muratori ne fait que prendre son développement dans le passé pour l'actualiser dans le présent. La pensée subit une accélération redoutable: il ne faut plus chercher dehors des modèles abstraits entachés de simplification et grossièrement mensongers. La ville, toujours actuelle et contemporaine, est un processus

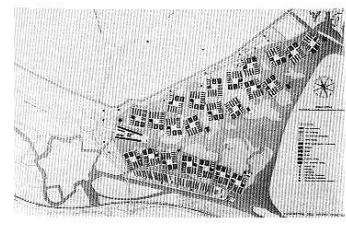

Trois plans pour les Barene di San Giuliano à Mestre (1959) présentent trois temps de la formation du tissu : autour de I'an mille (Estuario I, mention

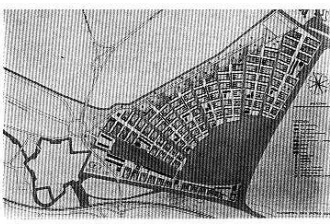

honorable), période gothique (Estuario II) et, basée sur le xvIII siècle, celle « qui paraît moderne " (Estuario III, primée).

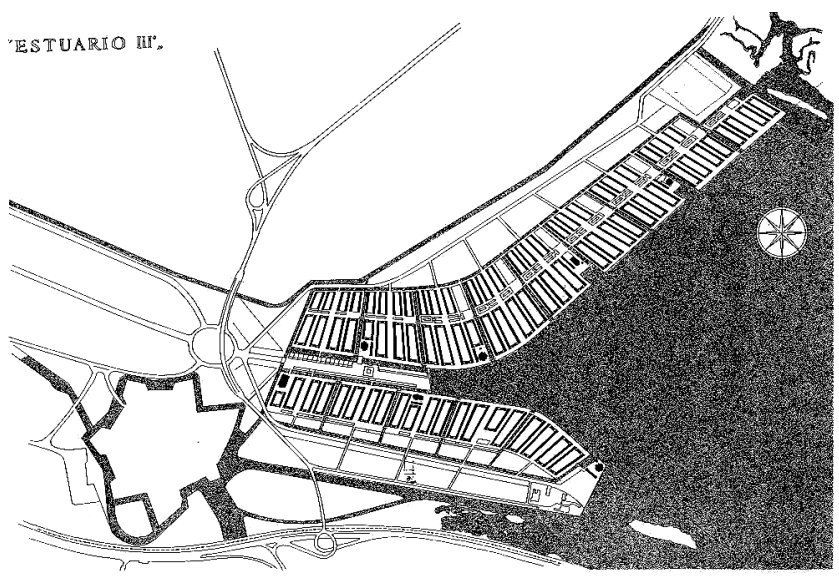




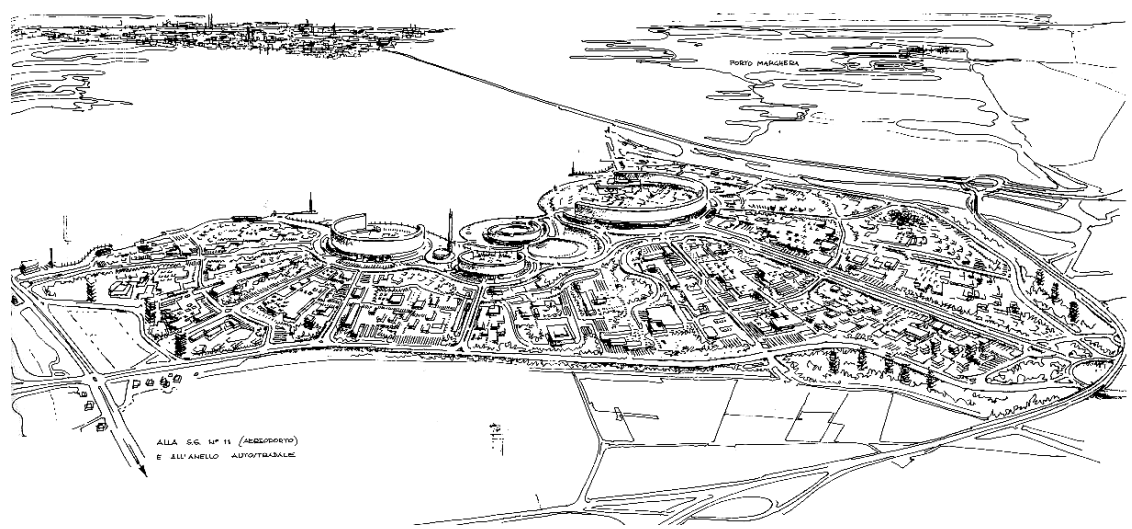

\begin{abstract}
Ludovico Quaroni exprime pour les Barene di San Giuliano l'acceptation totale d'un plan rationnel, opposé à Venise sur l'horizon.
\end{abstract}

historique qui s'accomplit dans le présent. Dans ce processus, connu par le vécu, les origines sont proches et s'enchaînent pour fournir une forme et des types à travers lesquels la ville d'aujourd'hui saura s'accomplir. Muratori a observé Venise, il a réuni un répertoire de solutions et il propose " une pure et simple reconduction du passé " (Caniggia). Le projet s'est aussitôt heurté à un barrage redoutable qui a empêché sa réalisation. Caniggia qui, en 1959, devient l'assistant de Muratori, était convaincu des qualités « impensables pour les architectes d'alors ». II nous revient d'en faire le procès: donnons la parole aux critiques, puis laissons passer 25 ans, I'hostilité n'a pas disparu, bien que d'intelligentes réalisations aient appliqué la méthode de Muratori au tissu vénitien en 1981-1989.

\section{L'impossible compréhension du projet de Muratori}

Le concours des Barene di San Giuliano, rendu en août 1959 par 200 équipes, est le seul proposé par le ministère des Travaux publics. II dépasse la taille d'un quartier autonome, avec ses 50000 habitants. Le concours est fait pour séduire: en plus des 38,50 hectares du quartier résidentiel, les candidats se voient offrir une surface le long de la côte, d'une « ample » respiration pour assurer les liaisons entre la terre ferme et la lagune. Le vainqueur sera chargé de la rédaction du plan d'urbanisme et sera flanqué des sept autres mentionnés qui se partageront la réalisation. Sauront-ils s'entendre? Giovanni Astengo (Urbanistica $n^{\circ} 31$, juillet 1960) en doute. II vient de montrer que les sept mentionnés présentent des

22. Dans son «Viatico alle psicopatie lagunari », Bruno Zevi, L'Architettura, n 57, juillet 1960, donne un résumé en français d'où sont extraites les citations.

23. Leonardo Benevolo mériterait une longue étude par sa position d'architecte et d'historien. Convaincu de la nécessité d'expliquer le Mouvement moderne dans une Italie qui l'avait rejeté, il livre des ouvrages essentiels sur l'architecture de la Renaissance, de l'époque moderne, sur 
solutions " riches d'intérêt [...] animées d'une forte idée motrice", mais "seules les solutions de Muratori s'en séparent, et le concours risque d'être un combat intime et irréductible entre les conceptions du coordinateur d'un côté et des coordonnés de l'autre » (p. 95).

La querelle relayée par la revue L'Architettura de juillet 1960 oppose Bruno Zevi et Leonardo Benevolo dans le numéro 242 de Casabella à une recherche plus savante sur la notion de type montrée peu avant par I'historien Giulio Carlo Argan dans le Bollettino del Centro Internazionale de Studi di Architettura Andrea Palladio, vol. I, 1959. Quelle que soit I'admiration que I'on prête à Bruno Zevi, son éditorial «Viatique pour les psychopathies lagunaires » le révèle prêt à trancher avec brutalité: il manie la plume comme un instrument de guerre. Le résumé écrit en français, en marge, aligne les motifs de condamnation ${ }^{22}$ : " position absurde ", " copier les monuments majeurs est immoral, copier les dialectes est à la fois ridicule et impossible », "la rhétorique de "l'architecture mineure » est délétère et artificieuse comme le plus mauvais éclectisme au $x x^{e}$ siècle. »

La fureur du débat révèle autre chose qu'un vague assentiment. Bâtir une « ville satellite de type rationnel, flanquée d'un vaste parc sur la lagune », voilà comment suivre la lignée de l'urbanisme moderne. II applaudit au projet de Quaroni, "plastiquement éloquent», parce qu'il dresse sur la lagune, face à Venise, "l'enveloppe de hauts cylindres ouverts jusqu'à la mer ». II condamne Muratori parce qu' « une copie du Parthénon engendre I'obscénité, mais décalquer une maison en bande de Burano est simplement ridicule ». II ne lui trouve " aucune idée, aucune méthode, aucun principe ", sinon la suprématie de l'esthétique vernaculaire qui prévaut sur l'économie et sur les mœurs contemporaines.

Poussant une "guerre sainte sans merci », Benevolo 23 qui a la charge de quatre pages dans le numéro 242 de Casabella ${ }^{24}$, "dépasse les limites de la polémique culturelle $»^{25}$. II rejette le vainqueur, Muratori, accusé de s'enfermer dans la pureté de sa solution, de refuser de négocier, de faire, par sa position extrême, vaciller tout espoir d'entente. Il est inadmissible, dans les années 1960, de maintenir un « concept aussi archaïque et conventionnel de milieu habité » en le vidant de tous « les systèmes complémentaires » qui, en plus de l'habitation, permettraient «sa vie et son développement harmonieux ». La faiblesse de Muratori vient de ce qu'il croit être sa force: le milieu habité sur lequel repose son discours est absent. Rien ne vaut de dresser aux Barene un tissu reproduisant la Venise ancienne, " avec ordre et régularité, face au chaos de Mestre ». Benevolo laisse soudain sourdre sa rupture d'avec Muratori, il ne lui accorde rien, il l'accuse d'être « comme Dracula, un vampire, qui ne peut circuler seul que jusqu'à l'aube, et autrement se désintègre ».

La position d'Argan manifeste à l'inverse une vision positive de la typologie. La fin de l'été 1959 avait permis de mettre face à face des architectes, des historiens de I'architecture et des historiens de l'art, à la villa CordelinaLombardi, à Montecchio Maggiore, à 12 kilomètres de Vicence, du 27 août au 16 septembre ${ }^{26}$. Sans omettre la définition de Quatremère de Quincy, Argan donne quatre leçons essentielles. Le type est «vague et générique », et pour mieux comprendre, il faut penser à la superposition d'une série d'éléments qui fait disparaître les caractères spécifiques pour ne conserver
I'histoire de la ville entre 1968 et 1974. II pratique une architecture "moderne » et la défend avec une arrogance étonnante, dans des affrontements sans merci.

24. Casabella, $n^{\circ} 242$, juillet 1960, comprend une partie intitulée « II concorso per il quartiere residenziale alle Barene di San Giuliano, Venezia-Mestre », p. 32-52 qui inclut l'article de Benevolo « Un consuntivo delle recente esperienze urbanistiche italiane », p. 33-36. Les citations sont extraites de son texte.
25. Franco Purini, « La scuola romana dagli anni sessanta agli anni ottanta. Un'educazione sentimentale all'architetttura », dans M. Montuori (dir.), Lezioni di progettazione, op. cit. note 1. La remarque s'adresse aux adversaires de Muratori, je l'applique ici à Benevolo.

26. Giulio Carlo Argan, «Typologia, Simbologia, allegorismo delle forme architettoniche », p. 13-16 et Bruno Zevi, «Problemi di interpretazione critica dell'architettura veneta » et « Attualità culturale di Michele Sanmicheli », p. 70-74, dans Bollettino del Centro Internationale di studi di Architettura Andrea Palladio, vol. I, 1959. 
que ce qui fait l'unité de la série: un schéma où se révèle "la réduction d'une série de variantes formelles pour exprimer leur structure commune ». La typologie ne se réduit pas aux nécessités du classement, elle a un but " esthétique » et donc inventif. Quant aux fonctions, on ne doit pas commettre l'erreur de croire qu'elles sont reflétées par la forme, mais qu'elles traduisent leur configuration, par une souplesse que I'histoire démontre par des phénomènes de substitution. Argan connaît bien la menace qui pèse sur le XIX ${ }^{e}$ siècle, qui « rigidifie " la typologie, au risque de la "typification », appauvrissant la richesse de l'art, par types d'hôpitaux, d'hôtels, de banques, etc. II voit le danger de "déhistoriciser " l'architecture par cette conception étroite du type, par la «suspension du jugement historique » qui rend incapable de décréter la mort d'un type ancien, refusant alors de le remplacer par un type nouveau.

\section{Vingt-cinq ans après, toujours la guerre de tranchées sur le type}

Comparer le projet de la Giudecca de Gino Valle (1981-1984) et celui de l'ex-Saffa de Vittorio Gregotti (1984-1989) avec l'îlot type de Muratori pour les Barene (1959), révèle que la pratique a donné raison à Muratori. Le projet de Valle, qui complète sur la rive sud la reconversion de la brasserie Dreher, est la transcription d'un tissu de rues rapprochées qui découpent des îlots filiformes, fréquents à Venise. II en reprend même le retournement en fin d'îlots, qui laisse place à des palais "les pieds dans l'eau », changés ici en belles demeures urbaines. L'ensemble résidentiel de Gregotti ${ }^{27}$ propose le même type d'îlots. La reconnaissance de la ville à l'entour est complète, elle fait droit aux campi, campielli, aux portiques qui abritent la vie sociale. Le type "à cour" permet de donner aux habitants une hiérarchie de lieux offerts à la pratique, avec jardins, loges ou toits-terrasses poétiquement entremêlés, et d'évoquer l'identité vénitienne par l'emprunt à un langage facilement compréhensible. Les toits de tuile, les corniches, les terrasses protégées par des cages de bois, les escaliers saillants reprennent l'idée d'une culture populaire, avancée 30 ans auparavant, en 1954 pour le quartier INA-Casa de Tiburtino. Certaines esquisses préliminaires ne se cachent pas d'évoquer la bourgade rurale tancée par Aldo Rossi ${ }^{28}$.

Gregotti, intéressé par le territoire, comme l'était avant lui Muratori, était persuadé que l'analyse typologique devait y apporter sa preuve. En 1966, il avait donné sa propre définition du type: «Le concept de type vise à ordonner l'expérience selon des schémas qui la rendent opératoire (sur la plan de la connaissance et de la construction), en réduisant à un nombre fini de cas l'infinité des phénomènes possibles ${ }^{29}$. "

Le type est un ensemble de traits caractéristiques, susceptibles d'être imités. II n'est plus besoin de le remettre en cause: on peut le reproduire globalement, avec des réarrangements occasionnels. Le type fonctionne dans le projet comme une "boîte noire ". Boîte noire de Gregotti ou « type a priori » de Muratori, cherchons les différences. Muratori, impressionné par le débat philosophique, revient à l'épistémologie kantienne: la connaissance procède par a priori, pour le temps, l'espace et pour le «type ». Vite dit par S. Malfroy: "On a toujours le type avec soi ${ }^{30}$ »

27. Voir Ermanno Ranzani, « Gregotti Associati : Quartiere residenziale ex Saffa, Venezia », Domus, n 704, avril 1989

28. Aldo Rossi, avec Gian-Ugo Polesello et Francesco Tandori, dans Casabella, $n^{\circ} 241,1960$, traitent de la périphérie des villes modernes, p. 29-41. L'opinion qu'ils donnent du quartier de Tiburtino (de Quaroni et Ridolfi) surprend par sa dureté : ils n'y voient page $40 q^{\prime} u^{\prime}$ « une bourgade du latium du Moyen Âge » ou « un 
comme objet de connaissance et de repérage acquis par l'apprentissage. Le type, alors, n'est plus un produit de l'analyse, mais devient-il proche de la " boîte noire " qui s'impose au projet, par un processus d’héritage?

\section{Positions tranchées sur le type Werner Oechslin, l'intelligence du type centré sur la société}

Le débat sur le type, volontairement réduit ici, met face à face Werner Oechslin et Bruno Reichlin, devant Benevolo toujours hostile, Giancarlo De Carlo et Aldo Van Eyck effarouchés. Oechslin ${ }^{31}$ nous convainc qu' « un effort intellectuel minime» nous garantira d'opinions « voilées d'arrogance » que l'on trouve chez Zevi: « L'art est antitypologique, toute création architecturale est inévitablement l'interprétation individuelle de l'artiste, et c'est le style qui définit le type. " Oechslin s'étonne de l'importance que le débat italien donne au concept de type défini par Quatremère de Quincy et surtout de sa reprise par Giulio Carlo Argan ${ }^{32}$ en 1962. II se fait l'écho de malentendus dans la position d'Argan qui préfaçait un recueil de textes en I'honneur de Hans Seldmayer, sujet lui-même difficile. Oechslin préférerait qu'on fasse appel « à la distinction philosophique entre forme et matière, en proposant ainsi une correspondance conceptuelle entre type et figure ». Heinrich Wölfflin ${ }^{33}$ reprenait le schème kantien en distinguant le schème producteur et l'image produite: le schème est « une règle qui sert à déterminer notre intuition conformément à un certain concept général ». Caché dans la pensée, il est indispensable pour donner sens à l'intuition, et fait passer de l'a priori au monde sensible et contingent.
C'est dans cet esprit que Werner Oechslin modifie I'analyse de la notion de type chez Quatremère de Quincy: elle sera moins positive, parce qu'elle doit reconnaître le problème de l'application. Le type devient alors indispensable au projet. II faut revenir sur une lecture plus fine des vocables " architecture », "caractère " et "convention" de l'Encyclopédie méthodique de 1825, sans oublier les Considérations morales sur la destination des œuvres d'art de 1815 de Quatremère. Bien sûr, "il faut un précédent à tout ».

Le type s'ouvre à l'imitation. Par l'entremise de la géométrie, base vitruvienne de l'architecture ou par la médiation des sens, le type peut être représenté par un dessin, le dessin "architectonique », être transposé et utilisé. La conclusion de W. Oechslin mérite d'être citée en entier: "Il est évident qu'on ne peut plus traiter la typologie d'être un modèle simplificateur, qui standardise ou réduit l'architecture, si bien que nous devons y reconnaître une construction conduite avec intelligence, qui garantit la liaison des conditions à la fois systématiques autant qu'historiques et conventionnelles (et pour cela toujours centrées sur la société) qui tiennent l'architecture sous leur interdépendance réciproque. »

\section{Bruno Reichlin, la typologie du Moderne. Leonardo Benevolo, Giancarlo De Carlo et Aldo Van Eyck ${ }^{34}$, effarouchés par la typologie} Bruno Reichlin se donne, de son côté, le droit de montrer les bienfaits que l'étude typologique a introduit dans la pratique du projet moderne. Mais la typologie révèle quatre attitudes neuves: I'intelligibilité, la compréhension de l'architecture, la genèse de l'engendrement des types, gigantesque trompe-|'œil » par son caractère rural. Leur opposition à Muratori est totale, surtout pour la forme de la ville.

29. Vittorio Gregotti, II territorio dell'architettura, Milan, 1966, trad. fr., Le Territoire de l'architecture, Paris, L'Équerre, 1982, p. 95.

30. Interview de S. Malfroy par Martin Brière, 27 avril 1996, DEA, Henri Bresler (dir.),

Depuis Muratori, diversité et divergences à propos de la morphologie urbaine, Projet architectural et urbain, université Paris VIII et école de Paris-Belleville, 1996.

31. Werner Oechslin, «Per una ripresa della discussione tipologica », Casabella, $n^{\text {os }}$ 509-510, p. 66-73, en italien, avec un résumé en anglais.

32. Giulio Carlo Argan, «Sul concetto di tipologia architettonica », Progetto e destino, Milan, II Saggiatore, 1962.
33. Heinrich Wölfflin, Prolégomènes à une psychologie de l'architecture, Paris, Éditions Carré, 1996, avec une introduction de Bruno Queysanne [éd. originale allemande, Prolegomena zu einer Psychologie der Architektur, Munich, 1886]. 


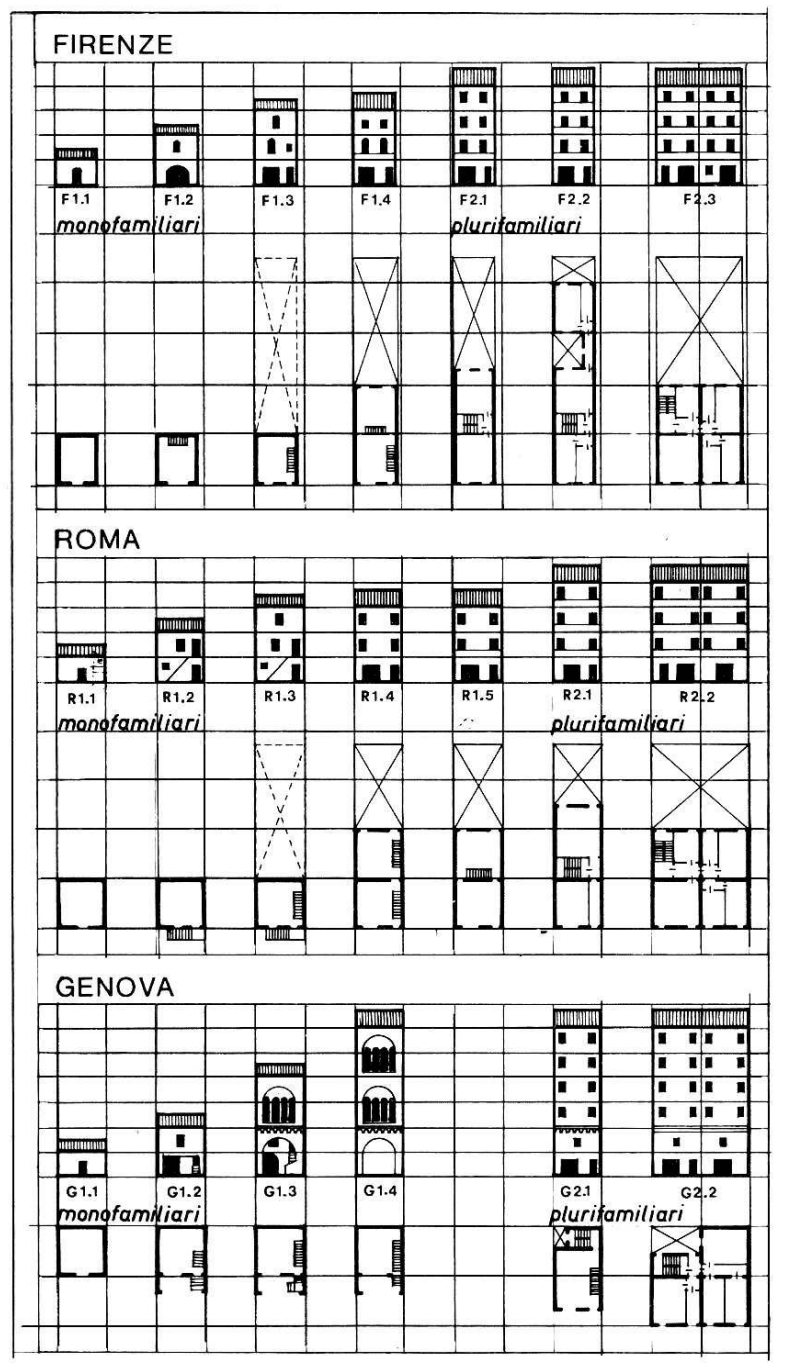

Mutations du type de base à Florence, Rome et Gênes. Gianfranco Caniggia, Gian Luigi Maffei, Lettura dell'edilizia di base, Venise, Marsilio, 1979.

Planche 12, p. 101.
Comprendre le territoire doit venir avant la ville. Les parcours de crête descendent à mi-pente et achèvent leur ramification.

Gianfranco Caniggia, Gian Luigi Maffei, Lettura dell'edilizia di base, Venise, Marsilio, 1979. Planches 52 et 53 , p. 208-209.

Dessins de Martinelli.
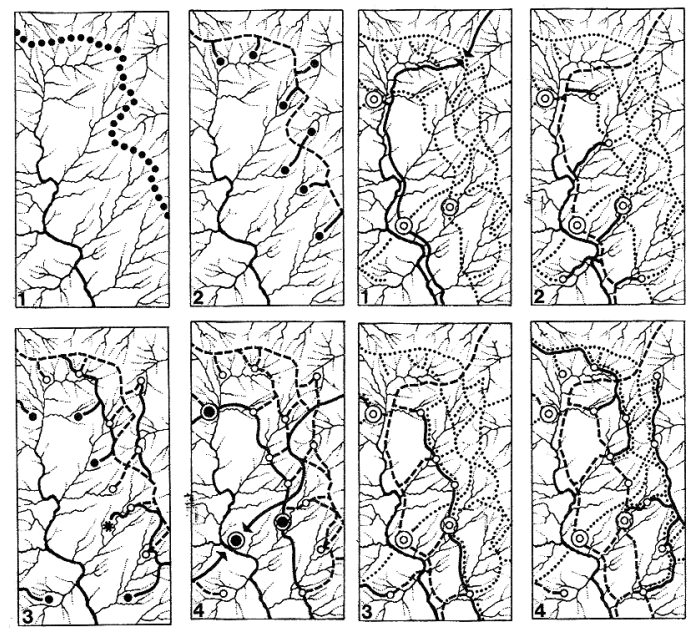

34. Qu'on ne cherche pas ici une critique de deux grands architectes, dont les travaux ont été célébrés à Urbino et aux Pays-Bas. Le choc des mots les renvoie du côté de Zevi ou de Benevolo sur la typologie. Tous deux membres de Team $X$ m'avaient posé des difficultés à cause de ma pratique de la typologie et de son usage dans le projet.

35. Le séminaire du Laboratoire international d'architecture et de design urbain, l'ILAUD, avait reçu une communication de Giancarlo De
Carlo en août 1979. L'analyse de la polémique a été ici fortement réduite. Voir Casabella, $\mathrm{n}^{\text {os }}$ 509-510, op. cit. note 30 , p. 46-51.

36. L'analyse typologique et la "typification » font passer du "type consacré » au "plan type » avec l'avènement de la Révolution industrielle. Philippe Panerai s'en explique clairement dans Philippe Panerai, Jean-Charles Depaule, Marcelle Demorgon, Analyse urbaine, Marseille, Éditions Parenthèses, 1999, p. 109-114.
La revue Casabella, nos 509-510, 1985, publie une contribution de Vittorio Magnago Lampugnani, «Typologia e tipizzazione. Typology and typification », p. 84-87.

37. Pier Giorgio Gerosa dans « Éléments pour une histoire des théories de la ville comme artefact et forme spatiale, XvIII siècleXIX siècle ", La ville comme artefact : la question typologique et la morphologie, Strasbourg, BfÉditions, 1990, donne un condensé sur l'école muratorienne, p. 178-194. 
surtout, l'innovation. Quatremère de Quincy, il fallait s'y attendre, approuvait par sa maxime: «Le type (permet à chacun) de concevoir une œuvre qui ne ressemble à aucune autre. " Alors, Reichlin fait face à la production des Modernes, depuis les années 1920. Sa vision optimiste propose une intelligence meilleure de la typologie de la production moderne, il fait œuvre d'ouverture, n'a rien à reprocher à Quatremère sur les degrés de transformations qui assurent la modernité du type.

Pour Leonardo Benevolo, la cause du type est entendue. Pour consommer la fin du classicisme, « il faut abandonner pour un temps toutes les références à la typologie »: la rhétorique classique est morte, la dialectique la remplace en cherchant « des règles, des méthodologies disciplinaires, des types », il refuse de manipuler d'anciennes traditions détachées de toute réalité historique. Le vieux type est mort. Benevolo ne croit qu'en la recherche patiente. Giancarlo De Carlo se donne pour effarouché par la typologie dans un message aux étudiants du laboratoire international d'architecture et de design urbain, ILAUD ${ }^{35}$ publié dans Casabella en août 1979, sous le titre « Notes sur l'ascèse débauchée de la typologie ». II reprend la conception frileuse de Gregotti, que le type n'est qu'un stéréotype, un type rigide, confondant typologie et typification ${ }^{36}$. Van Eyck, s'effraie de la dispute sur la typologie, la querelle des définitions, se retranche derrière un refus de comprendre. Son message à Gregotti le raidit: «Mon cher Vittorio, je n'ai jamais mis d'aussi sales mots sur du papier - et je n'ai jamais consenti à ce genre $d^{\prime \prime \prime o l o g i e " ~ o u ~ " o l o g i q u e ", ~ s i ~ b i e n ~ q u e ~ j e ~ n e ~ p e u x ~ p a s ~}$ faire partie de ta chambre d'horreurs intellectuelles. »

\section{La synthèse muratorienne}

Ces résistances, douze ans après la mort de Muratori, nous obligent à un retour en arrière, jusqu'aux années 1949-1973. Le champ européen est encombré par la remise en cause des dogmes des CIAM: le discours dominant est tenu par les membres de Team $X$, la situation en Italie n'échappe pas à des moments de contestation, I'assurance que prend Muratori sur I'analyse de la forme urbaine le conduit à livrer ses deux ouvrages sur Venise et sur Rome. L'école muratorienne se renforce, avec notamment Paolo Maretto, Gianfranco Caniggia, Gian Luigi Maffei et Giancarlo Cataldi. II convient donc d'affirmer les convictions accrues de l'école muratorienne pour faire valoir les solutions nouvelles de la pensée de la ville et de l'histoire « active » inspirées par Muratori.

\section{Le type comme concept actif ou l'adaptation graduelle de la ville}

Les assistants de Muratori font siennes ses hypothèses, les adaptent progressivement pour développer la théorie morphologique et en accroître l'efficacité. Ce qui exige de revenir sur les bases de sa pensée. Il fallait préciser la notion de type. Muratori s'était contenté « d'en donner des aperçus contextuels, tout au long de ses ouvrages ${ }^{37}$ ». Gerosa résume la définition réarrangée par Maretto après son travail sur Venise ${ }^{38}$. Le type ne résulte pas d'une analyse mais est connu de manière intuitive a priori: "Le type est donc une synthèse a priori, préexistante à l'acte de bâtir, une expression catégorielle totale, synthétique des aspects moraux, économiques, structuraux, technologiques, plastiques-
38. Paolo Maretto, L'edilizia gotica veneziana, Istituto Poligrafico Dello Stato, Libreria Dello Stato, 1960, repris dans P. Maretto, La casa veneziana nella storia della città, Venise, Marsilio Editori, 1986.

39. P. G. Gerosa, La Ville comme artefact, op. cit. note 36, p. 186.

40. Je réutilise le travail de DEA de Martin Brière, Depuis Muratori, diversité et divergences à propos de la morphologie urbaine, dirigé par Henri Bressler, Paris, école d'architecture de Paris-Belleville, 1996.

41. Gianfranco Caniggia, Ragionamenti di tipologia : operatività della tipologia processuale in architettura, Florence, Alinea, 1997, avec une introduction de Gian Luigi Maffei et un résumé en anglais de Nicola Marzot.

42. Henri Raymond, «Habitat, modèles culturels et architecture », dans L'Architecture d'aujourd'hui, no 174, 1974 et dans

L'Architecture, les aventures spatiales de la raison, Paris, Éditions du Centre Pompidou, 1993, p. 59, précise : «L'ensemble des modèles culturels [...] apparaît effectivement à la population comme ce qui reste stable, face à ce qui change [...], conditions conjoncturelles, économiques et sociales. " Raymond affirme que l'on vend « aujourd'hui sous les espèces de valorisation de classe [des maisons Phénix] qui diffèrent peu des lotissements d'Athis-Mons d'entre les deux guerres ». 
figuratifs. Cette catégorie universelle qu'est le type n'est pas une entité statique mais elle est ouverte à un développement directionnel continu caractéristique d'une certaine civilisation $^{39}$. $"$

Mais si le type est un concept actif, encore faut-il indiquer comment il se modifie. Caniggia ${ }^{40}$ a inventé le terme de processus typologique (en italien typologia processuale ${ }^{41}$ ). Il était gêné par l'emploi du mot de morphologie, car pour lui la forme est un leurre qui renvoie au type, structure cachée déterminante. C'est donc aux caractères typiques de se modifier, c'est eux qui portent sens. Quel est le rythme de ses transformations? II est fort lent et ne se voit que dans la longue durée. Caniggia observe que la ville ne progresse que par des adaptations graduelles qu'il pare du joli nom de " capillaires", faites de légères transformations dans des édifices stables. Leurs stabilités portent la trace d'un substrat culturel, par l'emploi d'un terme emprunté à la géographie et à la philosophie. Si le type se modifie par de modestes adaptations, il devient "type porteur" (typo portante en italien) en usant d'un terme où on voit bien que l'on a besoin de l'ancien pour trouver du

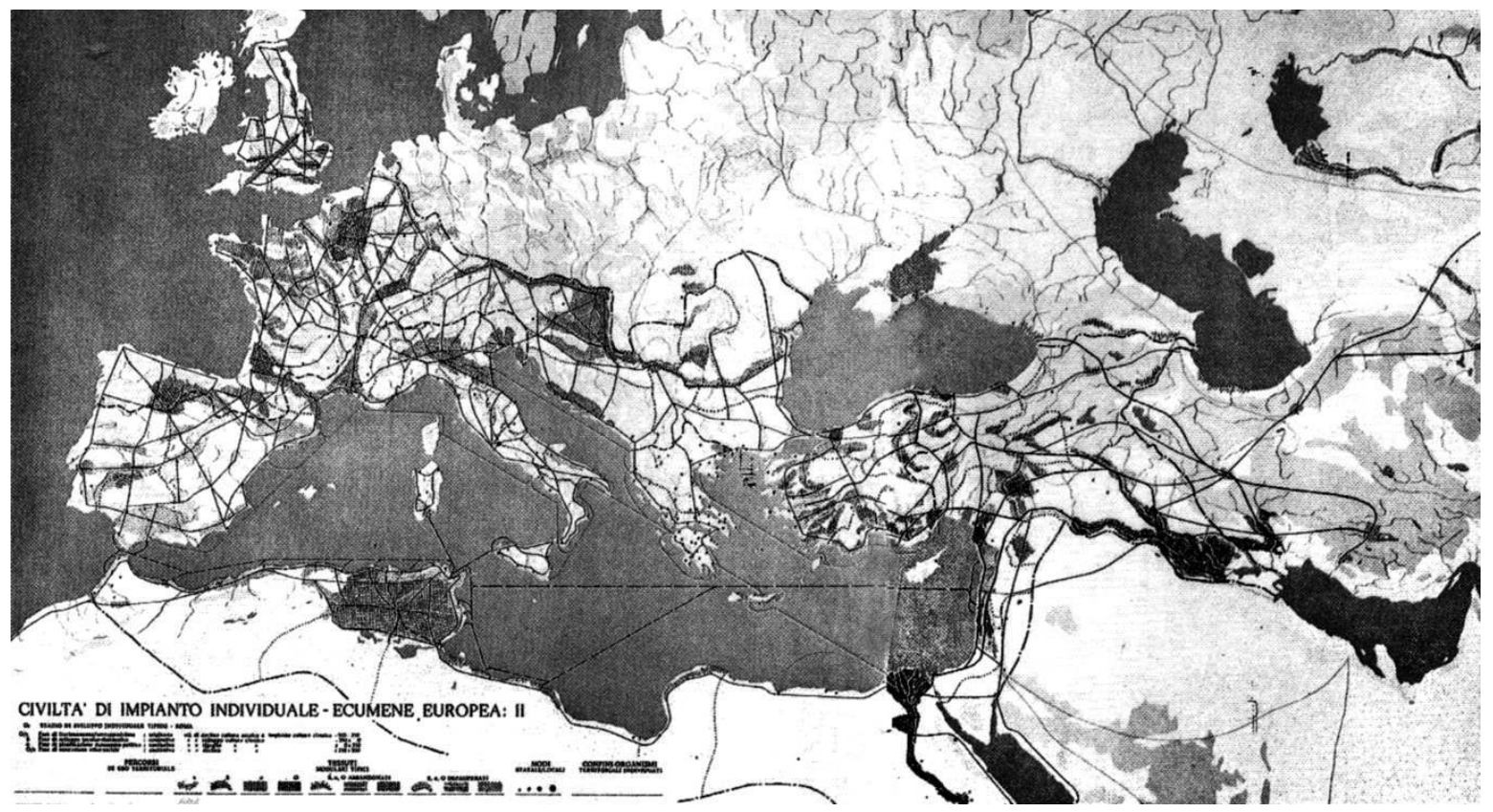

Schéma du monde romain et mésopotamien antique. Études faites entre 1969 et 1973 . Giorgio Pigafetta, Saverio Muratori architetto. Teoria e progetti, Venise, Marsilio, 1990. Planche 30. 
nouveau. Ce type peut se révéler par force obsolète, mais son substrat servira "de support à l'innovation d'un nouveau type porteur ».

Tout le travail mené aux Barene di San Giuliano s'éclaire précisément. Mais aussi la crise de la culture qui obsède Muratori et qui justifie les critiques lancées contre lui. Le besoin d'assurer la permanence des modes de construction heurtait les critiques, mais plus encore leur incompréhension de la lente transformation des «modèles culturels ${ }^{42}$ ». Elle s'oppose à l'inverse à l'idée trop commune de la mutation ${ }^{43}$ brutale que dément I'analyse sociologique ${ }^{44}$ surtout en France. Si I'habitat est une expérience formatrice d'habitudes, il s'agit moins de malmener les habitus des populations à traiter, mais de leur fournir de légères possibilités d'adaptation à une nouvelle situation dans le temps. La continuité du passé argumente pour le présent et pour l'avenir. Les positions françaises des années 1970-1980 prouvaient une grande compréhension de la théorie muratorienne, malgré la gêne due à la faiblesse des échanges qui dressaient une muraille entre l'Italie et la France. Mais empruntant fréquemment à Carlo
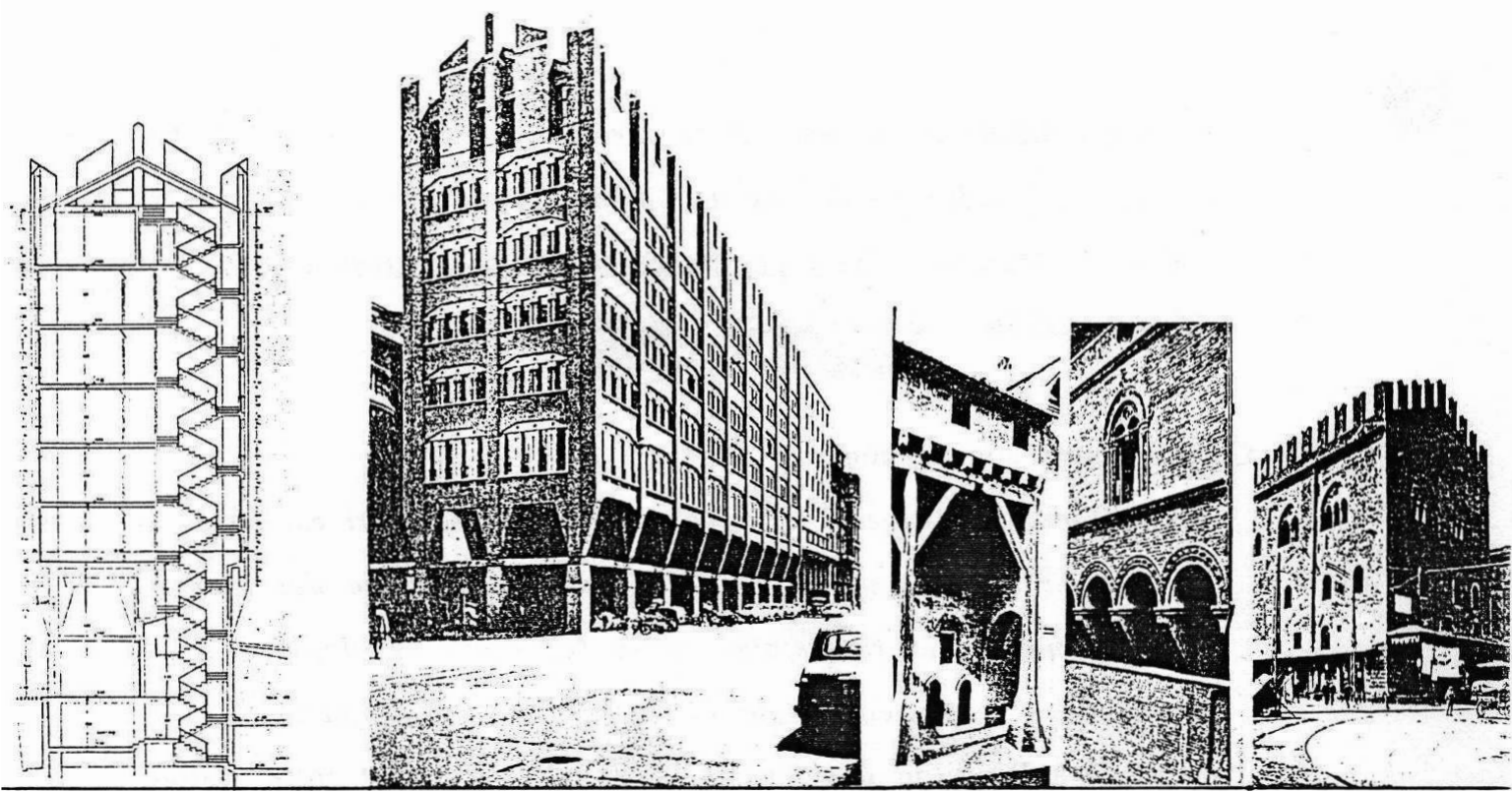

Le siège de

I'ENPAS à Bologne

regarde du côté

du gothique

mais réalise

une architecture

« moderne-

antique ".

Archives

Bollati-Marinucci.
43. Mutation veut dire «brusque et spontanée » et non graduelle.

44. Voir Jean-Charles Depaule, "Situation de I'analyse typo-morphologique en France, I'acquis de l'anthropologie », dans J. Castex, J.-C. Depaule, J.-L. Cohen, Histoire urbaine, anthropologie de l'espace, Paris, CNRS éditions, 1995.

45. Paolo Maretto, Realtà naturale e realtà costruita, Florence, Uniedit, 1980. 
Aymonino et à Aldo Rossi, certains se coupaient de l'orthodoxie muratorienne, en dissociant le projet de I'analyse, présentant de Muratori une version entachée de « moderne».

\section{La série des échelles}

De la parcelle au territoire, la synthèse muratorienne touche la série des échelles, appelée en italien gradi scalari ou traduite par Gerosa par «progression d'échelons ». Paolo Maretto a formalisé les échelles en $1973^{45}$. Le parler courant préfère dire maisons, rues, villes, régions. Elles s'ancrent dans la culture locale de ceux qui «parlent et habitent des maisons non projetées construites sans l'aide du dessin, proches d'eux et de leur milieu " qui avaient la faveur de Caniggia ${ }^{46}$. Nos façons savantes poussent à adapter les noms: parcellaire, îlot, territoire, et à trouver pour chaque niveau une typologie correspondante. La même démarche a été reprise par des anthropologues, sociologues, historiens modernes et géographes qui ont coordonné L'Aventure des mots de la ville (2010)"47: "Les mots de tous les jours » étaient choisis à la différence « de ceux qui appartiennent aux langages techniques, administratifs ou savants. " Ils excluent "les concepts abstraits », cherchant à montrer que « les mots de la ville ne font pas que décrire le monde urbain, ils contribuent à le constituer » (Depaule, Topalov, 1996). Pour Maretto, en 1973, qui refuse de nommer la forme urbaine, il dira " types de ville » et pensera à l'articulation des niveaux du bâti de l'élément au territoire, traversant le tissu et la ville. Dépendant toujours de Benedetto Croce, il divise la globalité en quatre séries d'échelle et montre comment passer de l'une à l'autre. Typologie parcellaire, éléments de liaison, d'association, le tracé parcellaire et sa pratique fournissent une typologie de cours, de rues qui deviennent la base de l'analyse de la ville, formes de tissus urbains, types de ville.

Rappelons-nous de la leçon de Muratori, tirée de Studi per un operante storia urbana di Venezia (1960): "Le type ne se caractérise pas en dehors de son application concrète, c'est-à-dire en dehors du tissu. » « Le tissu urbain à son tour ne se caractérise pas en dehors de son cadre, c'est-à-dire en dehors de l'étude de l'ensemble de la structure urbaine. " La fin de la vie de Muratori

\section{Le siège de la \\ Démocratie chrétienne \\ à Rome (1955-1958) \\ montre quelle \\ créativité est donnée \\ au passage du passé \\ au présent : voûtes,

\begin{abstract}
porte-à-faux des façades. Giorgio Pigafetta, Saverio Muratori architetto. Teoria e progetti, Planche 24.
\end{abstract} Venise, Marsilio, 1990.}

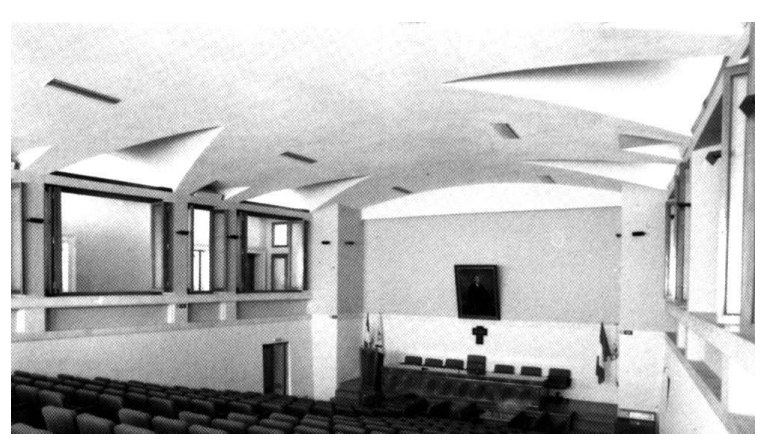

46. J. F. Caniggia, «Permanenze e mutationi nel typo edilizio e nei tessuti di Roma, 1880-1930 », dans G. Giuseppe Strappa, Tradizione e innovazione nell'architettura di Roma Capitale, 1870-1930, Rome, Kappa, 1989 , p. 15.

47. Christian Topalov, Laurent Coudroy de Lille, Jean-Charles Depaule, Brigitte Marin, L'Aventure des mots de la ville, à travers le temps, les langues, les sociétés, Paris, Robert Laffont, 2010.

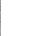 \\ .

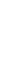


a été consacrée à généraliser ses observations pour atteindre l'objectif de traverser les continents pour y dégager « de grands organismes unitaires, typiquement différenciés entre eux ${ }^{48}$ où s'identifient les caractères propres des diverses cultures. Les planches des tabellone couvraient toutes les échelles, depuis les meubles, la distribution des appartements, les "schémas spatiaux " des édifices, les agrégations tissulaires, la forme des villes, les parcours qui structurent le territoire, par crêtes et vallées, l'étendue des mondes culturels, comme le monde romain, I'Inde ou la Chine.

Muratori faisait œuvre de structuraliste en reliant les structures «typiques » des établissements avec l'organisation "typique » des sociétés civiles. L'essentiel, pour lui, n'était pas de "tout réduire à un "concept", à un "lexique" ni à une "méthode" ", mais d'expliciter "la structure du réel ${ }^{49}$ " par un système qui apportait ses lois pour relier tous les éléments. L'intérêt porté au territoire lui permettait de « relier le tissu, les parcours et les structures orographiques", dans une logique reconnue par Manfredo Tafuri ${ }^{50}$. Surtout elle lui donnait la possibilité de projeter les expansions urbaines, sans que sa mort (en 1973) ne lui ait donné le temps de les réaliser ni même de publier ses résultats, qui restent sous forme d'enregistrements et de liasses de documents.

\section{Travailler l'histoire. La storia operante}

La réalité profonde de la pensée de Muratori était de solidariser le projet et l'analyse par une série de raisonnements qui actualisaient I'histoire: I'histoire c'est aujourd'hui, il n'y a pas de distance entre le jour d'aujourd'hui et le passé qu'elle accomplit. Si I'on a compris les trois étapes du projet des Barene di San Giuliano, c'est là que se fabrique le tissu actuel. Mais il s'agit du tissu anonyme de base qui renoue avec l'habitat spontané, faisant usage des techniques locales. La position de l'architecte doit être renversée. Cependant Muratori n'a pas craint, dans sa carrière d'architecte, d'édifier des "émergences " comme des églises, sièges sociaux d'un parti politique, la Démocratie chrétienne. Produire cette architecture demande finesse et complexité. Muratori désigne ce travail d'investigation typologique par le concept de «travailler l'histoire ».

Pour respecter les types, il faut limiter les ajouts successifs et refuser l'outrance individuelle. La position est éthique, elle se fonde sur la modestie de l'architecte qui n'a pas à faire œuvre individuelle, bien que, chaque fois, sa manière ne fasse que progresser. Le concept de storia operante vient de la pensée de Benedetto Croce en postulant non plus la force des oppositions mais la nécessité d'accorder les distinctions. Elle s'oppose aux excès de l'abstraction, postulant une « intuition transfigurée » par la posture de l'esprit qui relie les éléments distincts. Peut-être pourrait-on rendre storia operante par l'histoire à l'œuvre dans le présent, avec l'identité de l'analyse (appelée « lecture ») et du projet. Muratori est d'un historicisme absolu, il pense à l'histoire " comme pensée et comme action " comme le voulait Croce ${ }^{51}$.

Giorgio Pigafetta indique bien dans son Saverio Muratori architetto, le besoin d'éclairer une position claire dans la détresse de la production de l'architecture des années 1952-1958. Il fallait refuser à la fois le passéisme et la prétendue avant-garde. Le siège de
48. Giorgio Pigafetta, Saverio Muratori architetto, Venise, Marsilio, 1990, p. 151.

49. Ibid., p. 152.

50. M. Tafuri, Storia dell'architettura italiana, op. cit. note 29, p. 80 .
51. La phrase est tirée de G. Pigafetta, Saverio Muratori architetto, op. cit. note 48. 
I'ENPAS, à Bologne (1952-1957), sur une artère élargie et fréquentée, voie centrale, refaite au XIXe siècle, mais sur une partie détruite par la guerre, s'inspire des valeurs du gothique du centre de Bologne et réalise avec lui des transferts démonstratifs. Bâtiment de peu d'épaisseur, sur huit niveaux, il modernise le gothique, invente une modénature actuelle pour dénoncer la banalité de I'architecture du XIX siècle. Différent des projets anciens, il est la première expression de la ténacité avec laquelle Muratori tient à la storia operante. Le siège de la Démocratie chrétienne (1955-1957) est fondé sur la volonté de continuer l'architecture typique de Rome. Il réutilise le schéma du palais de la Renaissance du début $d u x v l^{e}$ siècle, il comprime au-dessous l'atrium, fait œuvre en respectant la distribution renaissante, invente des porte-à-faux pour placer le plan noble et faire déborder la toiture. Si la continuité $d u x v I^{e}$ siècle pouvait être convoquée en passant par-dessus le temps du Moderne, le palais a été jugé caricatural par Paolo Portoghesi avec l'excuse de l'intensité de la crise de I'architecture des années $1950^{52}$.

D'autres, comme Giannini ${ }^{53}$ le mettaient au niveau de " la meilleure architecture du siècle », mais une remarque de Franco Purini doit éveiller notre attention: «Les architectures de Muratori sont faites à travers lui », à la différence de Libera où elle est « faite par lui », comme si le fossé de l'histoire venait d'être effacé. Muratori se dresse contre un faux historicisme ${ }^{54}$, refuse la coupure entre le passé - le temps où la ville s'est faite - et l'action de construire au présent. L'histoire disparaît dans l'actualité du présent qui la poursuit: simplement, l'histoire est au présent, "à l'œuvre», sans rupture, capable de

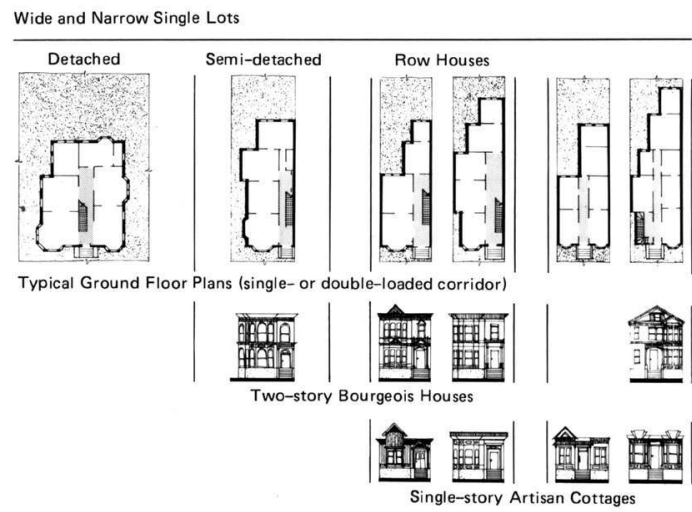

Single and Double Lots

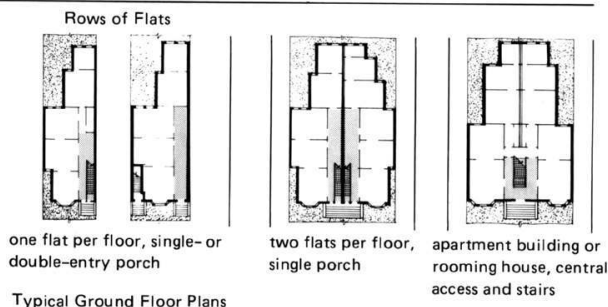

3.13

Typology of house

forms and lots

the nineteenth

century

Anne Vernez Moudon, dans Built for Change. Neighborhood Architecture in San Francisco, Cambridge (MA), The MIT Press, 1986, suit les enseignements de Caniggia. Les maisons victoriennes, basées sur la ventilation des pièces centrales, influencent Mac Donald dans son immeuble de 18 mètres de façade marqué par la tradition de la côte Pacifique (1979) : le « processus typologique " prend sa source dans les types anciens par de " graduelles adaptations ". Planches 3.13, p. 61 et E.11, p. 212.

52. Paolo Portoghesi, Dopo I'architettura moderna, Rome, Laterza, 1980, p. 83.

53. Me basant sur G. Pigafetta, Saverio

Muratori architetto, op. cit. note 48.

Pigafetta cite Giannini, L'ambiente,

p. 48 et Franco Purini, «Un'educazione

sentimentale all'architettura », dans Lezioni

di progettazione, op. cit. note 1, p. 288.

54. Ibid., discussion p. 89-90. 


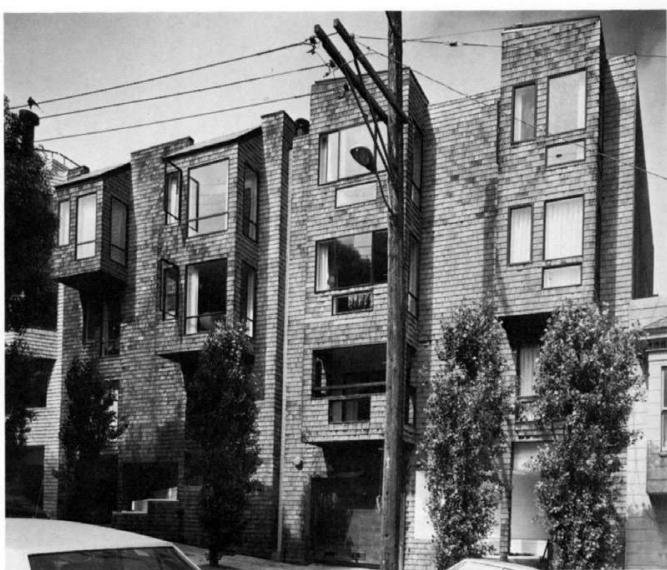

Rear Yard

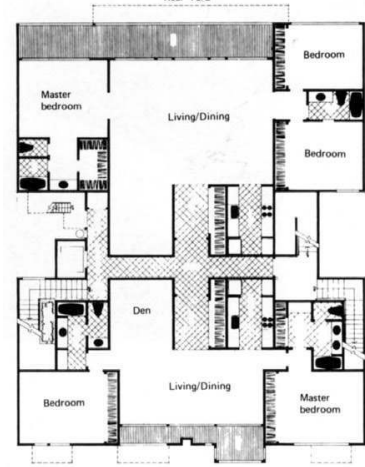

c

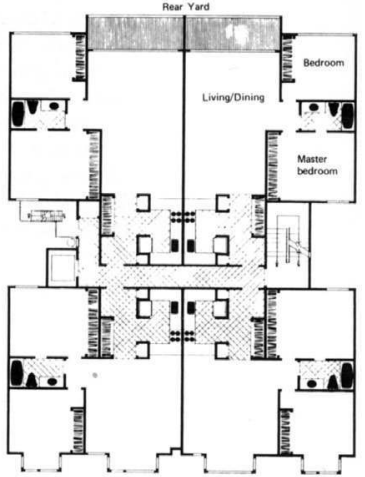

d

négocier l'aujourd'hui dans la reconnaissance d'un passé toujours actif. II ne peut y avoir qu'une vision unitaire qui crée, par-dessus les oppositions, les vecteurs efficaces de la continuité visible pour la vie des sociétés traduite dans les établissements urbains qu'il faut lire comme le lieu d'apprentissage des mœurs et de la vie quotidienne.

Le souci de traiter des "émergences" poussait à l'extrême les convictions de Muratori. II faut regarder avec soin le Palazzo Sturzo, siège de la Démocratie chrétienne. Il faut comprendre le mélange d'une ossature moderne et ses capacités d'expression classique, voir le rythme des fenestrages amplifié par des travées à la Serlio, tout en respectant les fenêtres en longueur d'inspiration corbuséenne. Surtout, les salles refusent les plafonds anonymes pour être voûtées par des réseaux de pénétrations qui dénoncent la misère de bien des bâtiments contemporains. Les porte-àfaux débordants sont réalisés en brique, portés par des arceaux qui prennent au-dessus des poutres en béton une lisibilité manifeste et incitent à un processus créatif qui rend l'histoire actuelle.

\section{Le tissu banal de la ville}

$\mathrm{Si}$ les "émergences» se comprennent comme des exceptions, bien qu'elles naissent du tissu de la ville, le tissu banal devait exciter les muratoriens. Je n'en prendrai qu'un exemple. Sous la direction de Giuseppe Strappa, les articles de Tradizione e Innovazione nell'architettura di Roma Capitale, 1870-193055 couvraient les moments de crise de la fin du XIX $x^{e}$ siècle et de l'émergence du "moderne » avant 1930. Caniggia y posait le problème de «la ville réelle», celle qui a été construite, et voulait

55. Giuseppe Strappa (dir.), Tradizione e Innovazione nell'architettura di Roma Capitale, 1870-1930, Rome, Kappa,

1989 : textes de G. Caniggia, P. Marconi, G. Strappa, L. Toschi. 
en restituer I'histoire en refusant les critères de sélection des historiens "modernes». II la révèle avec Giulio Magni (1859-1930), architecte important mais auquel on refuse sa place au premier rang ${ }^{56}$. II se spécialise dans l'architecture domestique, ses commandes abondent en immeubles urbains, bureaux, maisons à loyer, villas, et il dessine également des lotissements. II ne s'agit pas d'opposer ceux qui connaissent le mouvement européen et ceux qui l'ignorent. Mais de mettre le doigt sur un choix critique fait plutôt d'expériences autochtones, qui s'inscrit dans le lieu et qui demande d'exclure certains comportements pour n'accepter que «les valeurs déclarées sans opposition au bâti romain ». Tout son intérêt est d'approfondir l'architecture de Rome pour en tirer des solutions capables de résoudre le dramatique problème de l'extension urbaine née après l'unité de l'Italie.

L'article de Caniggia «Permanenze e mutazioni nel tipo edilizio e nei tessuti di Roma, 1880-193057 " prend garde de ne pas suivre l'accusation " odieuse et rituelle " que cette architecture ne fait qu'obéir aux dogmes de l'Académie. Faire un retour au $x v l^{e}$ siècle marque la différence entre les monuments (ou "émergences », églises, couvents, palais) qui constituent le discours « lettré » des architectes et la construction du bâti courant qui "parle » le langage simple utilisé par «d'humbles exécutants» non architectes. Cette juxtaposition donne sa consistance au tissu de Rome: elle tient compte des lotissements du Champ de Mars, du Borgo, des axes de Sixte-Quint. La maison banale construite en bandes, sur deux ou trois étages, associée aux "émergences " suffit à faire la ville. Une relative fixité se poursuit jusqu'aux années 1880 .
Rome capitale, après 1870, connaît de vastes lotissements en grille, souvent coupés de diagonales, avec des îlots de 100 mètres par 60 ou 80 mètres que I'on trouve sur l'Esquilin, contre la gare Termini, ou de l'autre côté du Tibre, face au Champ de Mars, quartier des Prati ou de la place Mazzini. Critiqués comme monotones, ils sont dessinés par des architectes qui ne font que reprendre ce que les architectes $d u x v I^{e}$ au $X V I^{e}{ }^{e}$ siècle faisaient: ils copient des palais, pour abriter la bourgeoisie arrivante à Rome ou même pour loger des ouvriers.

Que les styles varient est de peu d'importance, palais $\mathrm{du} \mathrm{XVl} l^{\mathrm{e}}$ siècle, pittoresque médiévisant ensuite, faveur ré-accordée au Baroque des années 1920, nommée barocchetto. Mais les types d'habitation varient peu, se référant toujours à la maison en bandes vieille de trois siècles que l'on incorpore au schéma du palais: la seule nouveauté se trouve dans le groupement non plus de maisons familiales mais des appartements qui les remplacent, "qui ouvrent à une vaste gamme de solutions ». L'importance due aux revenus et la distance entre les classes étirent les solutions depuis les intensivi les plus denses jusqu'à des versions simplifiées des villas, appelées villini ou à la solution imaginative de la palazzina qui regroupe autour d'une ou de deux cages d'escaliers des piles d'appartements au contour diversifié. Villini et palazzine perdent ce que Caniggia nomme "l'aire de pertinence », l'espace externe associé à la maison, pour former "une sorte de vert semi-public unifié " commun à l'îlot qui se trouve ouvert de tous côtés. On trouvera bien sûr des variantes mixtes qui permettront de créer autour des villini des espaces communs de service et de passage, appelés du joli nom

56. Giulio Magni a beaucoup construit à Bucarest de 1894 à 1904, a été membre de l'Académie de Saint-Luc et a occupé le premier poste de professeur d'éléments constructifs quand il fut créé à l'école d'architecture de Rome de 1921 à 1930 
de chiostrina. La densité des intensivi (où le logement se limite à une salle et une cuisine) et l'aisance des palazzine (où la bourgeoisie profite de la division entre les pièces de jour et celles de nuit, avec entre les deux, cuisine et salle de bains) donnent un nouveau caractère à la ville de Rome au début du $x x^{\mathrm{e}}$ siècle. Caniggia ne refuse pas d'évoquer les chantiers d'INA-Casa de l'après-guerre et de s'employer à rétablir la continuité du bâti romain en dessinant des solutions de maisons en bandes, à la place de l'hôpital de la Trinità dei Pellegrini.

Cette remise en place du «tissu courant » à l'exclusion des ruptures qu'introduisent la modernité et I‘histoire " moderne », offre une vision réaliste de l'édification de la ville et prouve, avec Caniggia, le succès de l'école de Muratori. En étroite relation avec les quatre parties du texte sur l'école muratorienne, vient à l'esprit un livre savant sur I'histoire architecturale d'une ville: la Rome de la Renaissance. Paolo Portoghesi ${ }^{58}$ sent le besoin de faire comprendre la formation du tissu du Borgo et du Champ de Mars à Rome, avec des éléments d'archives qui fournissent l'idée du tissu de la ville. Anne Vernez Moudon I'a superbement montré dans son Built for Change. Neighborhood Architecture in San Francisco ${ }^{59}$, où elle maîtrise la permanence et le processus des modifications des maisons prises dans le tissu banal de la ville. À l'école de Versailles et à I'université de Versailles-Saint-Quentin, Alexis Markovics a réalisé une thèse sur "La fortune critique d'une production ordinaire: les immeubles parisiens d'Albert Joseph Sélonier, 1858-192660". Profondément ancrée dans le tissu de Bordeaux, la rue EugèneTénot, évoquée par Chantal Callais conte «L'Histoire d'une rue ordinaire ${ }^{61} »$. Lotir et construire des maisons mitoyennes en rez-de-chaussée et des « échoppes » fait voir, derrière «l'apparence de l'individuel » la présence du collectif. Mais la variation locale des types - changement de l'escalier, passage de porte cochère, ajout du garage - montre la souplesse et les capacités de renouvellement qu'illustrent les vastes lotissements de Bordeaux. Pensons ici, à Delft même, aux travaux de Susanne Komossa, de Han Meyer, de Max Risselada, de Sabien Thomaes et de Nynke Jutten sur l'Atlas of the Dutch Urban Block ${ }^{62}$, qui révèle les continuités entre le passé du $\mathrm{XVII}{ }^{\mathrm{e}}$ siècle et la production du tissu de la ville actuelle aux Pays-Bas.

L'étonnant succès de Muratori est dans cette ouverture disciplinaire. Mais si grande que soit cette réussite, si heureuse qu'ait été la postérité de Muratori, il reste deux exigences à accomplir. Savoir correctement affirmer les points précis de sa méthode. D’autre part, puisque ces résultats sont peu connus et que des morceaux essentiels qui permettaient à Muratori de généraliser ses observations en les poussant à la dimension du monde sont devenus inaccessibles, il faut en accélérer la connaissance. La faute en revient sans doute à l'absence de traductions. L'époque actuelle reste toujours menaçante, se dresse contre le banal, évite la ville, flatte les vedettes de l'architecture, mène toujours la guerre contre la typologie au nom de la liberté créatrice des architectes: la lutte de Muratori contre ses adversaires de 1960 est toujours au programme. Le savoir scientifique y perd ses critères d'analyse qui, dans un monde aujourd'hui en voie de métropolisation, renforceraient ses capacités d'interprétation et de conviction.
58. P. Portoghesi, Roma del Rinascimiento, vol. 2, Milan, Electa, s.d. (avant 1973).

"Documenti catastali » et « Analisi tipologica », p. 535- 590.
59. Anne Vernez Moudon, Built for Change. Neighborhood Architecture in San Francisco, Cambridge (MA), MIT Press, 1986.

60. Alexis Markovics, « La fortune critique d'une production ordinaire : les immeubles parisiens d'Albert Joseph Sélonier, 1858-1926 » dans fabricA, n², Versailles, Ladrhaus, 2008, p. 6-25.
61. Chantal Callais, « Histoire d'une rue ordinaire, la rue Eugène-Ténot à Bordeaux ", dans fabricA, n० 3, Versailles, Ladrhaus, 2009, p. 156-213.

62. Suzanne Komossa, Han Meyer, Max Risselada, Sabien Thomaes, Nynke Jutten, Atlas of the Dutch Urban Block, Bussum, Thoth Uitgeverij, 2005. 